Mon. Not. R. Astron. Soc. 000,17?? () $\quad$ Printed 27 November $2019 \quad$ (MN LTEX style file v2.2)

\title{
Kinematical Investigation of Possible Fast Collimated Outflows in Twelve Planetary Nebulae
}

\author{
J.S. Rechy-García ${ }^{1}$, M.A. Guerrero ${ }^{1}$, S. Duarte Puertas ${ }^{1}$, Y.-H. Chu ${ }^{2}$, \\ J.A. Toalá ${ }^{3}$, \& L.F. Miranda ${ }^{1}$ \\ ${ }^{1}$ Instituto de Astrofísica de Andalucía, IAA-CSIC, Glorieta de la Astronomía s/n, 18008 Granada, Spain. \\ ${ }^{2}$ Institute of Astronomy and Astrophysics, Academia Sinica (ASIAA), Taipei 10617, Taiwan, Republic of China \\ ${ }^{3}$ Instituto de RadioastronomíÄ́śa y Astrofísica (IRyA), UNAM Campus Morelia, Apartado postal 3-72, 58090 Morelia, Michoacan, Mexico
}

Last updated ; in original form

\begin{abstract}
A significant fraction of planetary nebulae (PNe) exhibit collimated outflows, distinct narrow kinematical components with notable velocity shifts with respect to the main nebular shells typically associated with low-ionization compact knots and linear or precessing jet-like features. We present here a spatio-kinematical investigation of a sample of twelve PNe with morphologies in emission lines of low-ionization species suggestive of collimated outflows. Using archival narrow-band images and our own high-dispersion long-slit echelle spectra, we confirm the presence of collimated outflows in Hen 2-429, J 320, M 1-66, M 2-40, M31, and NGC 6210 and possibly in NGC 6741, for which the spatio-kinematical data can also be interpreted as a pair of bipolar lobes. The presence of collimated outflows is rejected in Hen 2-47, Hen 2-115, M 1-26, and M 1-37, but their morphology and kinematics are indicative of the action of supersonic outflows that have not been able to pierce through the nebular envelope. In this sense, M 1-66 appears to have experienced a similar interaction between the outflow and nebular envelope, but, as opposed to these four PNe, the outflow has been able to break through the nebular envelope. It is suggested that the PNe without collimated outflows in our sample are younger or descend from lower mass progenitors than those that exhibit unambiguous collimated outflows.
\end{abstract}

Key words:

planetary nebulae, ISM: jets and outflows

\section{INTRODUCTION}

Planetary nebulae $(\mathrm{PNe})$ are the final stages of the life of lowand intermediate-mass stars $\left(1-8 \mathrm{M}_{\odot}\right)$, before they become white dwarfs. As such stars evolve through the asymptotic giant branch (AGB), they experience episodes of heavy mass-loss through a dense and slow AGB wind. Once the stellar core is exposed, these stars leave the AGB and their effective temperatures increase while they develop fast and tenuous stellar winds. The increasing UV stellar flux will ionize the slow AGB wind to give birth to a PN.

A large number of PNe exhibit fast collimated outflows, typically described as distinct narrow kinematical components with notable velocity shifts with respect to the main nebular shells. Examples abound in the literature: IC 4634 (Guerrero et al.|2008), Hu 2-1 (Miranda 1995), NGC 7354 (Contreras et al. 2010), KjPn8 (López et al. 1995), NGC 6337 (Corradi et al. 2000), and M 1-32 (RechyGarcía et al.2017). The reader is referred to our recent compilation and statistical investigation of collimated outflows in PNe (Guerrero et al.2019).
Collimated outflows are commonly associated with lowionization compact knots and linear or precessing jet-like features (Gonçalves et al.2001), but the sample of collimated outflows has diverse spatio-kinematical properties, which may point to different populations with different origins (Guerrero et al. 2019). Since collimated outflows in $\mathrm{PNe}$ are thought to play notable effects in their shaping and even formation (Sahai, \& Trauger 1998), a critical spatio-kinematical assessment of the nature of PN features classified as possible collimated outflows based on their morphologies and excitation conditions is most necessary.

In this paper, we present long-slit high-dispersion spectroscopic observations of a sample of PNe whose morphologies, as determined from narrow-band images, are indicative of the presence of collimated outflows. The spatio-kinematical information derived from these observations along the position angle (PA) of the possible collimated outflow has been used to confirm (or reject) their nature and to derive their systemic radial velocities. The sample of PNe and the observations are described in $\S 2$, the spatio- 
kinematical data of each PN is analysed in $\S 3$, and a discussion and a short summary are given in $\S 4$ and $\S 5$, respectively.

\section{OBSERVATIONS}

\subsection{Imagery}

Collimated outflows in PNe are best identified in narrow-band images of emission lines of low-ionization species of [N II], [O I], [O II], or [S II] (Gonçalves et al. 2001). We have selected a sample of PNe with narrow-band images in [N II], [O II] or $\mathrm{H} \alpha$ emission lines indicative of the presence of collimated outflows with no available kinematical data. The sample is presented in Table 1 Most sources have high-quality narrow-band images obtained with the Wide-Field Planetary Camera 2 (WFPC2) on board of the $\mathrm{Hub}$ ble Space Telescope (HST), except IC 4776, whose narrow-band image in the [O II] emission line was obtained with the instrument FORS2 at the Very Large Telescope (VLT) of the European Southern Observatory (ESO) in Chile (Sowicka et al. 2017), and Hen 2-429, whose narrow-band image in the [N II] emission line was obtained with the instrument ALFOSC at the Nordic Optical Telescope (NOT) of the Observatorio de El Roque de los Muchachos (ORM) on the island of La Palma in Spain (Manchado et al. 1996).

\subsection{Kinematical data}

Spatially-resolved high-dispersion echelle spectroscopic observations were obtained along a series of long-slit apertures placed across nebular features of interest. The Utretch Echelle Spectrograph (UES) on the $4.2 \mathrm{~m}$ William Herschel Telescope (WHT) of the ORM and the echelle spectrograph on the Cerro Tololo Interamerican Observatory (CTIO) 4m Víctor Blanco (aka Blanco) telescope were used for these observations.

The high-dispersion spectroscopic observations of the northern sources were obtained on 13 July 1995, 6 August 1996, and 10 December 1998 using UES at the WHT (Tab. 1). The spectrograph was used in its long-slit mode with a narrow-band filter that isolates the echelle order in the spectral range from 6530 to $6600 \AA$, thus including the $\mathrm{H} \alpha$ and $[\mathrm{N}$ II] $\lambda \lambda 6548,6583$ emission lines. A 79 line $\mathrm{mm}^{-1}$ echelle grating was also used. The data were recorded with the Tektronix CCD detector, providing a spatial scale of $0 .{ }^{\prime \prime} 36$ pixel $^{-1}$ and a sampling of $3.2 \mathrm{~km} \mathrm{~s}^{-1}$ pixel $^{-1}$ along the dispersion direction. The slit has an unvignetted length of $160^{\prime \prime}$ and its width was set to 1 !' 1 , resulting in an instrumental resolution of 6.5 $\mathrm{km} \mathrm{s}^{-1}$. The angular resolution, determined by the seeing, varied between 1 !' 1 and 2 !'2 (Tab. 1). Individual spectra have typical exposure times of $1,800 \mathrm{~s}$.

The high-dispersion spectroscopic observations of the southern sources were obtained on 24-25 December 2001 and 22-25 June 2002 using the echelle spectrograph on the CTIO Blanco telescope (Tab. 11. The spectrograph was also used in its long-slit mode with a narrow-band $F W H M \sim 50 \AA$ filter to isolate the echelle order including the $\mathrm{H} \alpha$ and $[\mathrm{N}$ II] $\lambda \lambda 6548,6583$ emission lines. The 79 line $\mathrm{mm}^{-1}$ echelle grating and the long-focus red camera were used, resulting in a reciprocal dispersion of $3.4 \AA \mathrm{mm}^{-1}$. The data were

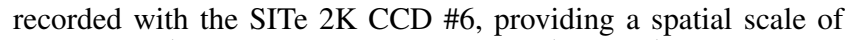
0 .' 26 pixel $^{-1}$ and a sampling of $3.7 \mathrm{~km} \mathrm{~s}^{-1}$ pixel $^{-1}$ along the dispersion direction. The slit has an unvignetted length of $3^{\prime}$ and its width was set to $0 . / 9$, resulting in an instrumental resolution of 8.0 $\mathrm{km} \mathrm{s}^{-1}$. The angular resolution, determined by the seeing, varied between $1 . " 3$ and 1.! 8 (Tab. 1). Individual spectra have typical exposure times of $1,800 \mathrm{~s}$.

The spectra were reduced using standard IRAF tasks for twodimensional spectra. The wavelength scale and geometrical distortion were corrected using a two-dimensional fit to arc exposures obtained using Th-Ar calibration lamps inmediately before or after the science exposure of each source. The deviation of the residuals of the two-dimensional fit to the Th-Ar arcs is found to be better than $0.004 \AA\left(0.2 \mathrm{~km} \mathrm{~s}^{-1}\right)$. The telluric emission, which includes the geocoronal $\mathrm{H} \alpha$ line, was removed by fitting and subtracting the background using low-order polynoms. The echelle observations were made with the slit oriented along different position angles and placed at various offsets from the central stars in order to sample different morphological features of interest (Tab. 1).

\section{RESULTS}

The narrow-band images and position-velocity (PV) maps of selected emission lines along PAs of interest are presented in Figures 1 to 12 using a hyperbolic sine inverted gray-scale. The images are overlaid with the location of the slits used to obtain kinematical information (Tab. 1). The brightest regions in the PV maps are shown saturated and overlaid with contours to display simultaneously information of the faintest and brightest features of these PV maps. These PV maps have been corrected to the Local Standard of Rest (LSR) velocity system and the spatial offsets have been measured from the central star or from its projection onto the slit.

The radial velocity in the LSR $\left(v_{\mathrm{LSR}}^{\text {sys }}\right)$ and heliocentric $\left(v_{\text {hel }}^{\text {sys }}\right)$ systems and nebular expansion velocity of these sources $\left(v_{\text {exp }}\right)$ are listed in columns 3 to 5 of Table 2 respectively. The radial velocities have been derived from the $\mathrm{H} \alpha$ emission line profiles of the main nebular shells at the location of the central star, whereas the profiles of the narrower [N II] $\lambda 6583 \AA$ emission line have been used to determine the nebular expansion velocities. When the emission line profile is resolved into receding (red-shifted) and approaching (blue-shifted) components, the systemic radial velocity is computed as the average between the centroid of both components and the nebular expansion velocity as their semi-difference. Typically, the velocity from an emission line can be determined with an accuracy $\sim 10 \%$ the spectral resolution, which combined with the wavelength calibration uncertainty of $0.2 \mathrm{~km} \mathrm{~s}^{-1}$, implies velocity uncertainties within $\pm 1.0 \mathrm{~km} \mathrm{~s}^{-1}$. In cases when the emission line profile is unresolved, the systemic radial velocity is computed as the centroid of the emission line profile and the nebular expansion velocity as the half width of the line profile once the instrumental and thermal widths have been substracted quadratically. The radial velocities of sources in Table 2 are generally in agreement $\left(\leqslant 10 \mathrm{~km} \mathrm{~s}^{-1}\right)$ with previous available measurements, as for Hen 2-115 ( $v_{\text {hel }} \simeq-56.2 \mathrm{~km} \mathrm{~s}^{-1}$, Durand et al. 1998), Hen 2$249\left(v_{\text {hel }} \simeq 30 \mathrm{~km} \mathrm{~s}^{-1}\right.$, Guerrero et al. 1999), IC $4776\left(v_{\text {hel }} \simeq 16.3\right.$ $\mathrm{km} \mathrm{s}^{-1}$ and $v_{\mathrm{LSR}} \simeq 27.9 \mathrm{~km} \mathrm{~s}^{-1}$, Durand et al. 1998; Maciel, \& Dutra 1992), J 320 ( $v_{\text {hel }} \simeq-25 \mathrm{~km} \mathrm{~s}^{-1}$, Harman et al. 2004), M 1-26 ( $v_{\text {hel }} \simeq-22 \mathrm{~km} \mathrm{~s}^{-1}$, Ortolani, \& Sabbadin 1985), M 1$37\left(v_{\text {hel }} \simeq 213 \mathrm{~km} \mathrm{~s}^{-1}\right.$, Richer et al. 2017), M 1-66 ( $v_{\text {hel }} \simeq 27.9$ $\mathrm{km} \mathrm{s}^{-1}$, Durand et al. 1998), M 2-40 ( $v_{\text {hel }} \simeq 90 \mathrm{~km} \mathrm{~s}^{-1}$, Beaulieu et al. 1999), M3-1 ( $v_{\text {hel }} \simeq 69.5 \mathrm{~km} \mathrm{~s}^{-1}$, Schneider et al. 1983), and NGC $6741\left(v_{\text {hel }} \simeq 41.3 \mathrm{~km} \mathrm{~s}^{-1}\right.$, Schneider et al. 1983). Only in Hen 2-47 the difference between the radial velocity in Table 2 and the heliocentric radial velocity of $-17.7 \mathrm{~km} \mathrm{~s}^{-1}$ reported by Durand et al. (1998) is larger than $10 \mathrm{~km} \mathrm{~s}^{-1}$.

The spatio-kinematical properties of their collimated outflows, 
Table 1. *

Sample and Observations of PNe with Possible Collimated Outflows

\begin{tabular}{|c|c|c|c|c|c|c|c|}
\hline \multirow[t]{3}{*}{ Source } & \multicolumn{3}{|c|}{ Imagery } & \multicolumn{4}{|c|}{ Spectroscopy } \\
\hline & \multirow[t]{2}{*}{ Telescope \& Camera } & \multirow[t]{2}{*}{ Band } & \multirow{2}{*}{$\begin{array}{l}\text { Resolution } \\
\left({ }^{\prime \prime}\right)\end{array}$} & \multirow[t]{2}{*}{ Telescope \& Spectrograph } & \multicolumn{2}{|c|}{ Resolution } & \multirow{2}{*}{$\begin{array}{l}\text { PA } \\
\left(^{\circ}\right)\end{array}$} \\
\hline & & & & & $\left(\mathrm{km} \mathrm{s}^{-1}\right)$ & $\left({ }^{\prime \prime}\right)$ & \\
\hline Hen 2-47 & $H S T$ WFPC2 & {$[\mathrm{N}$ II $]$} & $0 . " 1$ & Blanco Echelle & 8.0 & 1.4 & 0,64 \\
\hline Hen 2-115 & $H S T$ WFPC2 & $\mathrm{H} \alpha$ & $0 . " 1$ & Blanco Echelle & 8.0 & 1.4 & 9,275 \\
\hline Hen 2-429 & NOT ALFOSC & {$[\mathrm{N}$ II $]$} & 0.7 & WHT UES & 6.5 & 1.2 & 90 \\
\hline IC 4776 & VLT FORS2 & [O II] & $1 . " 0$ & Blanco Echelle & 8.0 & 1.7 & 45 \\
\hline J 320 & HST WFPC2 & $\mathrm{H} \alpha$ & $0 . " 1$ & Blanco Echelle & 8.0 & 1.8 & $306,338,348$ \\
\hline M 1-26 & HST WFPC2 & $\mathrm{H} \alpha$ & $0 .{ }^{\prime \prime} 1$ & Blanco Echelle & 8.0 & 1.5 & $38,82,325$ \\
\hline M 1-37 & HST WFPC2 & $\mathrm{H} \alpha$ & $0 . " 1$ & Blanco Echelle & 8.0 & 1.3 & 39,309 \\
\hline M 1-66 & $H S T$ WFPC2 & $\mathrm{H} \alpha$ & $0 . " 1$ & WHT UES & 6.5 & 2.2 & 311 \\
\hline M 2-40 & HST WFPC2 & $\mathrm{H} \alpha$ & $0 . " 1$ & WHT UES & 6.5 & 1.1 & 80 \\
\hline M3-1 & HST WFPC2 & [N II $]$ & $0 . " 1$ & Blanco Echelle & 8.0 & 1.8 & $72,318,358$ \\
\hline NGC 6210 & $H S T$ WFPC2 & $\mathrm{H} \alpha$ & $0 . " 1$ & Blanco Echelle & 8.0 & 1.8 & 312,336 \\
\hline NGC 6741 & HST WFPC2 & {$[\mathrm{N}$ II] } & $0 . " 1$ & Blanco Echelle & 8.0 & 1.3 & $18,77,302$ \\
\hline
\end{tabular}

including their identification, morphology, radial velocities with respect to the systemic velocity $\left(v_{\mathrm{r}}^{\text {outflow }}\right)$, and projected linear sizes $(\delta r)$ are listed in columns 6 to 9 of Table 2 respectively. Hereafter, we will refer to the radial velocity of the outflow with respect to the nebular systemic velocity as the systemic velocity. In most cases, kinematical information was available for both the approaching and receding outflow components, and thus the systemic velocity was derived as the semi-difference between the radial velocities of these two components.

\subsection{Hen 2-47}

Hen 2-47 (PN G285.6-02.7) is a young PN with a highly asymmetric multipolar morphology consisting of four pairs of V-shaped lobes and two rings with point-symmetric brightness distribution (Sahai 2000). Its shape is very similar to that of M 1-37 (see $\$ 3.7$ ) and both of them are consequently nicknamed the Starfish Twins. The complex shape of these young PNe has been proposed to result from the interaction of high-velocity collimated outflows with the nebular envelope in the late AGB or at the beginning of the postAGB phase (Sahai, \& Trauger 1998).

The kinematics of Hen 2-47 is investigated here using 9 slit positions oriented along $\mathrm{PA}=0^{\circ}$ and $64^{\circ}$ with different offsets from the central star of the PN (CSPN), as listed in Table 1. The most relevant [N II] $\lambda 6583 \mathrm{PV}$ maps are presented in Figure 1]

The expansion velocity of Hen 2-47, as derived from the emission profile of the $[\mathrm{N} \mathrm{II}]$ line in the slit across the central star along $\mathrm{PA}=64^{\circ}$ (not shown in Figure 1 is found to be $11.0 \mathrm{~km} \mathrm{~s}^{-1}$.

Following Sahai (2000)'s naming convention, features N2 and $\mathrm{S} 3$ are registered by the slit at $\mathrm{PA}=0^{\circ}$ across the CSPN, feature S2 is registered by the slit at $\mathrm{PA}=0^{\circ}$ and $3^{\prime \prime}$ East of the CSPN, and its counterpart N4 is registered by the slit at PA $=64^{\circ}$ offset by $2 .{ }^{\prime \prime} 5$ North from the CSPN. The PV maps indeed reveal that these features have kinematical properties differing from those of the main nebula. Multiple velocity components are detected wherever the slit intersects several V-shaped lobes, indicating that these have different radial velocities. From our data, we have been able to infer systemic velocities $v_{\mathrm{r}}^{\text {outflow }}$ of $\pm 23.5 \mathrm{~km} \mathrm{~s}^{-1}$ and $\pm 22.9 \mathrm{~km} \mathrm{~s}^{-1}$ for the pairs of features N2-S3 and N4-S2, respectively. Kinematically, these outflows do not look very collimated, in agreement with the impression derived from the $\mathrm{H} \alpha$ image. These features have pro- jected distances from the CSPN of $2.3 \times 10^{17} \mathrm{~cm}(0.074 \mathrm{pc})$ and $2.2 \times 10^{17} \mathrm{~cm}(0.071 \mathrm{pc})$, respectively.

\subsection{Hen 2-115}

Hen 2-115 (PNG321.3+02.8) is classified as an elliptical nebula (Stanghellini et al. 1993), although the HST WFPC2 H $\alpha$ image in Figure 2 reveals a complex morphology consisting of a bright bipolar structure around the central star and a pair of narrow Vshaped lobes protruding along PA $=95^{\circ}$ (Sahai, \& Trauger 1998), which seem indicative of the effects of a bipolar outflow. The main nebula is surrounded by a diffuse halo (Sahai et al. 2011).

The kinematics of Hen 2-115 is investigated using two slits oriented along its minor axis at PA $9^{\circ}$ and along its major axis and collimated outflows at PA $95^{\circ}$. The [N II] PV map of the first slit (not shown in Figure 2) has been used to derive a nebular expansion velocity $\simeq 13.4 \mathrm{~km} \mathrm{~s}^{-1}$.

The $[\mathrm{N} \mathrm{II}] \lambda 6584 \mathrm{PV}$ map along $\mathrm{PA}=95^{\circ}$ shown in Figure 2 reveals the presence of a low-velocity $\left(v_{\mathrm{r}}^{\text {outflow }}= \pm 5.9 \mathrm{~km} \mathrm{~s}^{-1}\right)$ component at the location of the V-shaped lobes. As for Hen 2-47, the outflow does not seem particularly collimated and the data presented here fail to detect emission from a compact knot expanding ballistically at the tip of the V-shaped lobe, e.g. as in CRL 618 (Balick et al.2013 2014). This feature has a projected distance from the $\mathrm{CSPN} \simeq 2 \times 10^{1 /} \mathrm{cm}(0.069 \mathrm{pc})$.

\subsection{Hen 2-429}

The [N II] image of Hen 2-429 (PN G048.7+01.9) in Figure 3 shows an elliptical shell and a pair of outer low-ionization pointsymmetric filaments (Guerrero et al. 1999, Gonçalves et al.|2001).

Our kinematical investigation uses two slits placed at PA = $80^{\circ}$ and $90^{\circ}$ across the central star. Since the point-symmetric outer filament is covered by both slits, only the [N II] $\lambda 6583$ PV map of the slit at $\mathrm{PA}=90^{\circ}$, registering emission at the tip of the filament, is shown in Figure 3 The nebular expansion of Hen 2-429 is well resolved in these PV maps. Its expansion velocity is found to be $\simeq 30.6 \mathrm{~km} \mathrm{~s}^{-1}$, in good agreement with that of $30 \mathrm{~km} \mathrm{~s}^{-1}$ reported by Guerrero et al. (1999).

The point-symmetric filaments are detected both in the $\mathrm{H} \alpha$ and [N II] PV maps as relatively bright and compact components 
Table 2. *

Spatio-kinematical Information of the Sample of PNe with Possible Collimated Outflows

\begin{tabular}{|c|c|c|c|c|c|c|c|c|}
\hline Source & $\begin{array}{c}\text { Distance }^{\star} \\
(\mathrm{kpc})\end{array}$ & $\begin{array}{c}v_{\mathrm{LSR}}^{\text {sys }} \\
\left(\mathrm{km} \mathrm{s}^{-1}\right)\end{array}$ & $\begin{array}{c}v_{\mathrm{hel}}^{\text {sys }} \\
\left(\mathrm{km} \mathrm{s}^{-1}\right)\end{array}$ & $\begin{array}{c}v_{\exp } \\
\left(\mathrm{km} \mathrm{s}^{-1}\right)\end{array}$ & Feature ID & Morphology & $\begin{array}{c}v_{\mathrm{r}}^{\text {outflow }} \\
\left(\mathrm{km} \mathrm{s}^{-1}\right)\end{array}$ & $\begin{array}{c}\delta r \\
(\mathrm{pc})\end{array}$ \\
\hline \multirow[t]{2}{*}{ Hen 2-47 } & \multirow[t]{2}{*}{3.8} & \multirow[t]{2}{*}{-16.3} & \multirow[t]{2}{*}{-4.0} & \multirow[t]{2}{*}{11.0} & N2-S3 & V-shape & 23.5 & 0.074 \\
\hline & & & & & N4-S2 & V-shape & 22.9 & 0.071 \\
\hline Hen 2-115 & 5.0 & -64.8 & -63.6 & 13.4 & $\mathrm{PA}=95^{\circ}$ & V-shape & 5.9 & 0.069 \\
\hline Hen 2-429 & 3.7 & +45.2 & +26.7 & 30.6 & $\mathrm{PA}=90^{\circ}$ & precessing outflow & 5.2 & 0.135 \\
\hline \multirow[t]{2}{*}{ IC 4776} & \multirow[t]{2}{*}{4.4} & \multirow[t]{2}{*}{+23.0} & \multirow[t]{2}{*}{+14.3} & \multirow[t]{2}{*}{$\ldots$} & A-B & bipolar lobes & 49.6 & 0.100 \\
\hline & & & & & C-D & outflow? & 84.1 & 0.174 \\
\hline \multirow[t]{3}{*}{ J 320} & \multirow[t]{3}{*}{5.8} & \multirow[t]{3}{*}{-44.4} & \multirow[t]{3}{*}{-29.8} & \multirow[t]{3}{*}{16.0} & $\mathrm{PA}=348^{\circ}$ & outflow & 19.9 & 0.276 \\
\hline & & & & & $\mathrm{PA}=338^{\circ}$ & outflow & 34.0 & 0.219 \\
\hline & & & & & $\mathrm{PA}=306^{\circ}$ & outflow & 24.8 & 0.104 \\
\hline \multirow[t]{2}{*}{ M 1-26 } & \multirow[t]{2}{*}{2.1} & \multirow[t]{2}{*}{-14.4} & \multirow[t]{2}{*}{-21.4} & \multirow[t]{2}{*}{$<7.0$} & $\mathrm{PA}=82^{\circ}$ & bow-shock & 38.2 & 0.038 \\
\hline & & & & & $\mathrm{PA}=145^{\circ}$ & bow-shock & 47.7 & 0.035 \\
\hline M 1-37 & 14.4 & +229.2 & +218.7 & 11.0 & $\mathrm{PA}=129^{\circ}$ & V-shape & 7.6 & 0.142 \\
\hline M 1-66 & 6.4 & +39.2 & +22.6 & 20.0 & $\mathrm{PA}=131^{\circ}$ & outflow & 7.0 & 0.106 \\
\hline M 2-40 & 5.4 & +98.9 & +82.8 & 17.6 & $\mathrm{PA}=88^{\circ}$ & outflow & 16.0 & 0.123 \\
\hline \multirow[t]{2}{*}{ M 3-1 } & \multirow[t]{2}{*}{4.5} & \multirow[t]{2}{*}{+46.3} & \multirow[t]{2}{*}{+65.6} & \multirow[t]{2}{*}{24.5} & $\mathrm{PA}=318^{\circ}$ & precessing outflow & 14.9 & 0.268 \\
\hline & & & & & $\mathrm{PA}=358^{\circ}$ & precessing outflow & 13.2 & 0.168 \\
\hline \multirow[t]{3}{*}{ NGC 6210} & \multirow[t]{3}{*}{2.1} & \multirow[t]{3}{*}{-26.7} & \multirow[t]{3}{*}{-45.7} & \multirow[t]{3}{*}{34.2} & A & precessing outflow & 19.5 & 0.084 \\
\hline & & & & & $\mathrm{B}$ & outflow & 30.6 & 0.045 \\
\hline & & & & & $\mathrm{C}$ & precessing outflow & 29.4 & 0.174 \\
\hline \multirow[t]{2}{*}{ NGC 6741} & \multirow[t]{2}{*}{3.2} & \multirow[t]{2}{*}{+56.9} & \multirow[t]{2}{*}{+40.2} & 23.4 & A-B & outflow & 22.7 & 0.126 \\
\hline & & & & & $\mathrm{C}$ & outflow & 7.3 & 0.100 \\
\hline
\end{tabular}

$\star$ Distances adopted from Frew et al. (2016,
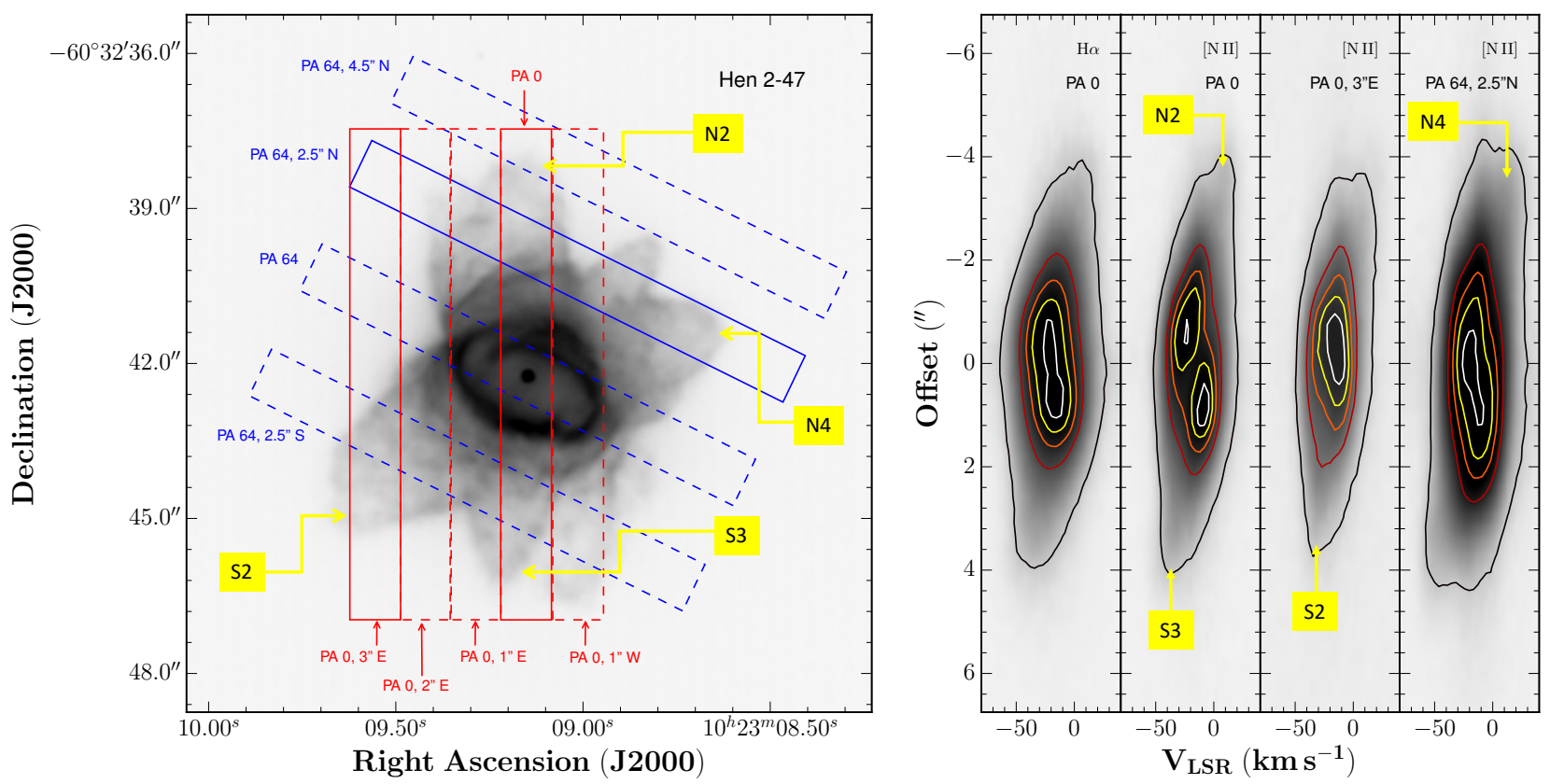

Figure 1. HST WFPC2 F658N [N II] image (left) and $\mathrm{H} \alpha$ and [N II] $\lambda 6583$ PV maps derived from CTIO 4m echelle spectra (right) of Hen 2-47. The names of the different morphological feature follow Sahai (2000). The positions of the slits are overlaid on the image on the left panel; the slits whose PV maps are shown on the right panels are drawn with solid lines, those not shown are drawn with dashed lines. 

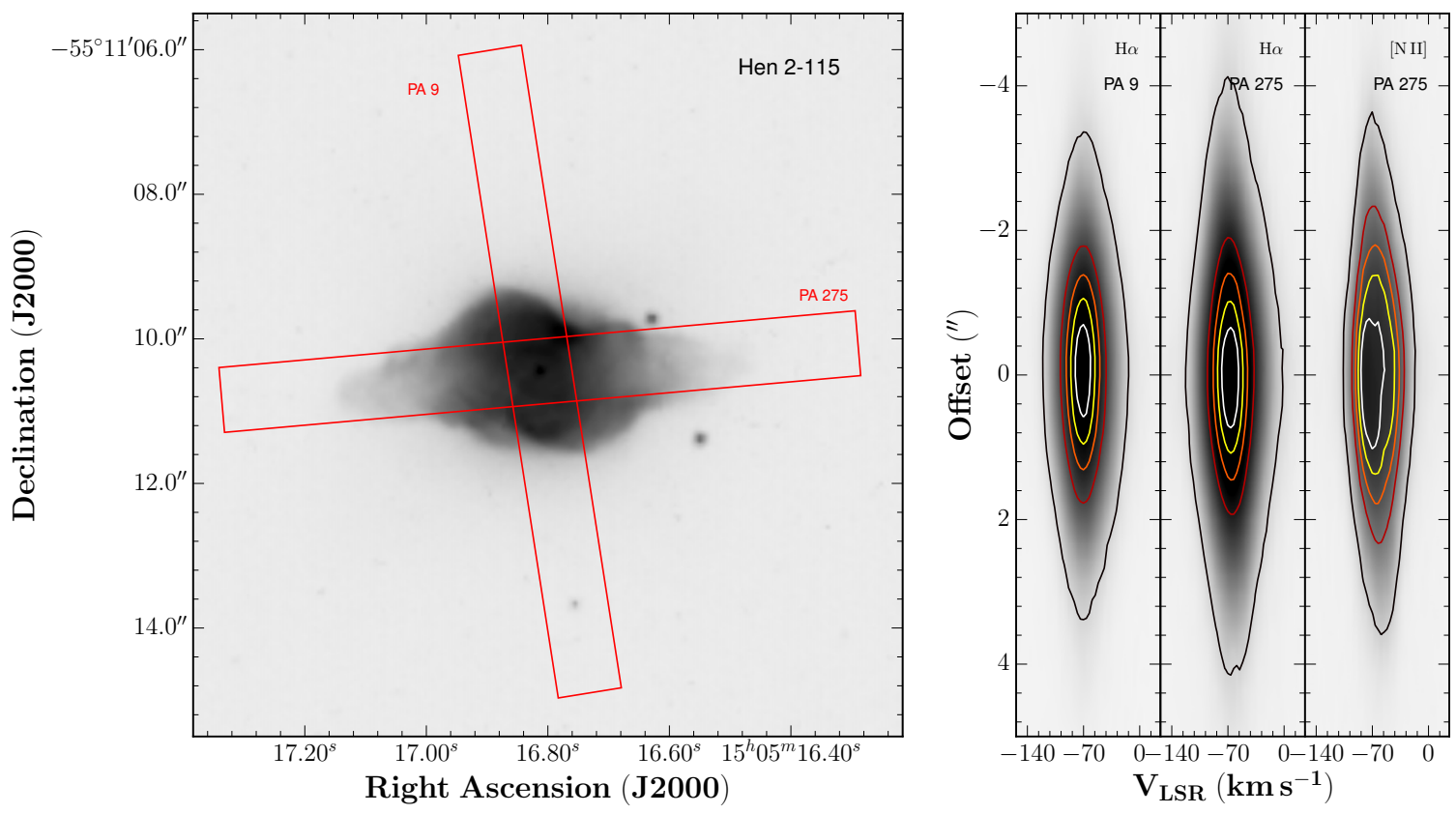

Figure 2. Same as Figure 1 for Hen 2-115. In this case, the $H S T$ image was obtained using the F656N H $\alpha$ filter.
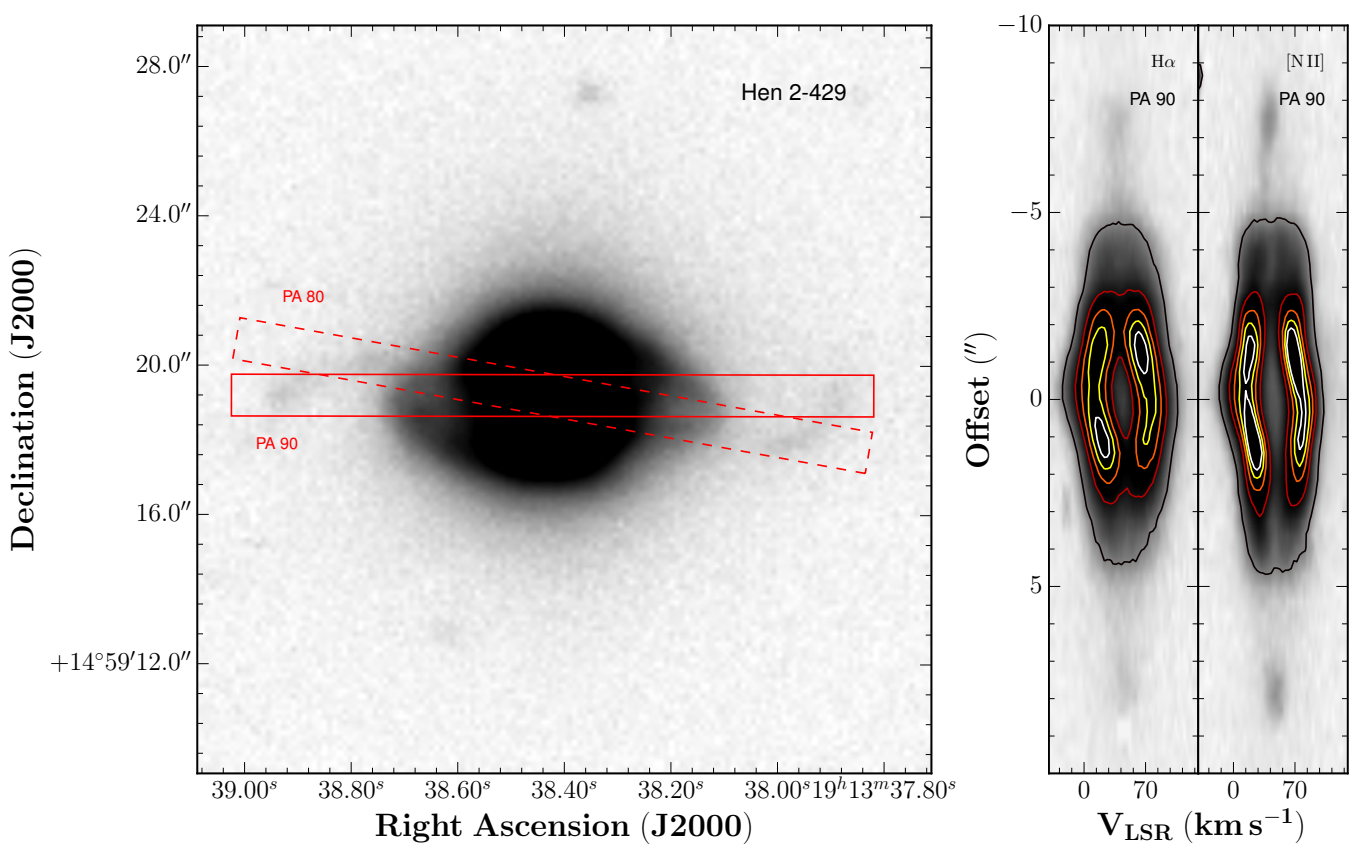

Figure 3. NOT ALFOSC [N II] image (left) and H $\alpha$ and [N II] $\lambda 6583$ PV maps derived from WHT UES echelle spectra (right) of Hen 2-429. Solid and dashed slits plotted on the image as in Figure 1

that are moving at a systemic velocity $\pm 5.2 \mathrm{~km} \mathrm{~s}^{-1}$. The kinematical properties of these filaments was already discussed by Guerrero et al. (1999), who noted their low systemic velocity. Interestingly, there is faint emission between these knots and the main nebula, suggesting these are the tips of bow-shock-like features. Their projected distance from the CSPN is $4.2 \times 10^{17} \mathrm{~cm}(0.14 \mathrm{pc})$.

\subsection{IC 4776}

Recent VLT FORS2 narrow-band imaging of IC 4776 (PN G002.0-13.4) in the $\mathrm{H} \alpha+[\mathrm{N} \mathrm{II}],[\mathrm{O}$ II], and [O III] emission lines have revealed an hourglass-shaped main nebula with its major axis aligned along $\mathrm{PA}=45^{\circ}$ and a pair of precessing jet-like features (Sowicka et al.2017).

Its kinematics is investigated here using a slit oriented along its major axis (Figure 4). The expansion velocity of the inner bright- 

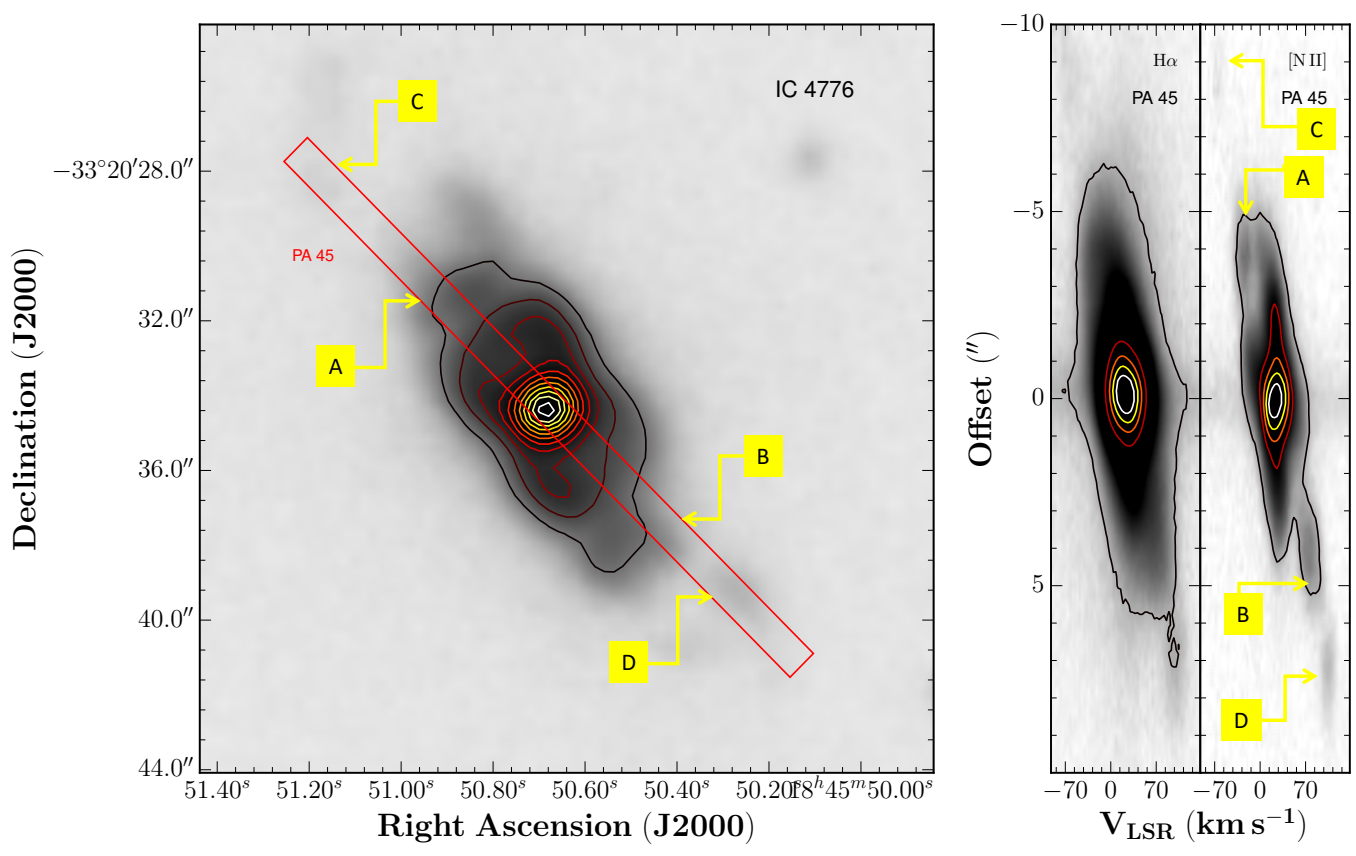

Figure 4. VLT FORS2 [O II] image (left) and $\mathrm{H} \alpha$ and [N II] $\lambda 6583 \mathrm{PV}$ maps derived from CTIO $4 \mathrm{~m}$ echelle spectra (right) of IC 4776.

est nebular regions is lower than $8 \mathrm{~km} \mathrm{~s}^{-1}$, in agreement with previous estimates $\left(\simeq 10.2 \mathrm{~km} \mathrm{~s}^{-1}\right.$, Bianchi 1992).

The $\mathrm{H} \alpha$ and $[\mathrm{N}$ II] $\lambda 6583 \mathrm{PV}$ maps reveal distinct structural components for the main nebula: an ellipsoidal shell in the $\mathrm{H} \alpha$ line, but an $\mathrm{X}$-shaped structure in the [N II] emission line. Four features named as A, B, C, and D are marked in Figure 4 as possible collimated outflows. The $\mathrm{X}$-shaped pattern is revealing of a bipolar PN whose symmetry axis is tilted with the line of sight. This interpretation is basically consistent with that provided by Sowicka et al. (2017), but the extent of the bipolar lobes of $\simeq 12^{\prime \prime}$ in size derived from our PV maps is larger than that of $\simeq 7^{\prime \prime}$ derived from the simulated PV map presented in figure 4 by Sowicka et al. (2017) because we interpret that the A and B features in our PV maps close the X-shaped pattern onto bipolar lobes. The systemic velocity of features $\mathrm{A}$ and $\mathrm{B}$ is found to be $\pm 52.2 \mathrm{~km} \mathrm{~s}^{-1}$. As for the outermost $\mathrm{C}$ and $\mathrm{D}$ pair of features, their systemic velocities are larger, $\pm 84 \mathrm{~km} \mathrm{~s}^{-1}$, and they look like compact knots, although we cannot discard they are the brightest regions of one additional pair of pointsymmetric bipolar lobes oriented along the same nebular axis (e.g., M 1-16 and M 2-9 Schwarz 1992, Schwarz et al. 1997, Clyne et al. 2015). These features have projected distances from the CSPN of $3.1 \times 10^{17} \mathrm{~cm}(0.10 \mathrm{pc})$ and $5.4 \times 10^{17} \mathrm{~cm}(0.17 \mathrm{pc})$, respectively.

\subsection{J 320}

J 320 (PN G190.3-17.7) is a spatially complex PN discovered by Jonckheere (1916) and classified morphologically as pointsymmetric (Gonçalves et al. 2001). It presents a bright elliptical inner rim, two bipolar lobes and several pairs of jet-like features oriented along different directions (Campbell, \& Moore 1917). The latter suggest that the ejecting direction is rotating, possibly with a time dependent velocity (López 1997).

To investigate the kinematics of $\mathrm{J} 320$, spectra were obtained using eight parallel slits oriented North-South and with offsets of $1^{\prime \prime}$ between positions (red dash boxes in Fig. 5-left) and four slits oriented at $\mathrm{PA}=40^{\circ}, 306^{\circ}, 338^{\circ}$ and $348^{\circ}$. The nebula has an expansion velocity $\simeq 16 \mathrm{~km} \mathrm{~s}^{-1}$, derived in this case from the $\mathrm{H} \alpha \mathrm{PV}$ maps across the CSPN.

The most interesting PV maps correspond to the slits at PA = $338^{\circ}$ and $348^{\circ}$ (Figure 5), which reveal that the bipolar lobes tips are bright pairs of knots. Following Harman et al. (2004)'s naming convention for these knots, we mark them in both the $\mathrm{H} \alpha$ image and PV maps in Figure 5 as $\mathrm{K} 1_{\mathrm{N}}$ and $\mathrm{K} 1_{\mathrm{S}}$ for the knots detected in the slit at $\mathrm{PA}=338^{\circ}$, and $\mathrm{K} 2{ }_{\mathrm{N}}$ and $\mathrm{K} 2_{\mathrm{S}}$ for the slit at $\mathrm{PA}=348^{\circ}$. The systemic velocity of these pairs of knots is $\pm 34.1 \mathrm{~km} \mathrm{~s}^{-1}$ for $\mathrm{K} 1$ and $\pm 19.9 \mathrm{~km} \mathrm{~s}^{-1}$ for $\mathrm{K} 2$, in agreement with the velocities reported by Harman et al. (2004). The slit at PA $=306^{\circ}$ reveals in the main body a pair of knots, one brighter than the other, with systemic velocity $\pm 24.8 \mathrm{~km} \mathrm{~s}^{-1}$. The projected distances from the CSPN of these features are $6.7 \times 10^{17} \mathrm{~cm}(0.217 \mathrm{pc})$ for the pair of knots $\mathrm{K} 1,8.5 \times 10^{17} \mathrm{~cm}(0.275 \mathrm{pc})$ for the pair of knots $\mathrm{K} 2$, and $3.2 \times 10^{17} \mathrm{~cm}(0.105 \mathrm{pc})$ for the pair of knots along $\mathrm{PA}=306^{\circ}$.

\subsection{1-26}

Whereas ground-based images of M 1-26 (PN G358.9-00.7) show an apparently featureless elliptical disk (Górny et al. 1997), the HST WFPC2 $\mathrm{H} \alpha$ image in Figure 6-left discloses it as a bright round shell surrounded by multiples loops and arcs (Sahai, \& Trauger 1998). The multipolar morphology of M 1-26, which makes it look like a rose, prompted Bear, \& Soker (2017) to include it among the sample of PNe likely shaped by triple stellar progenitors.

The kinematics of M 1-26 has been investigated using three long-slit apertures placed along the minor nebular axis at the PA = $38^{\circ}$, and at the location of outflow-like features along $\mathrm{PA}=82^{\circ}$ and $145^{\circ}$. The $\mathrm{H} \alpha \mathrm{PV}$ map for $\mathrm{PA}=38^{\circ}$ and $[\mathrm{N} \mathrm{II}] \lambda 6583 \mathrm{PV}$ maps 

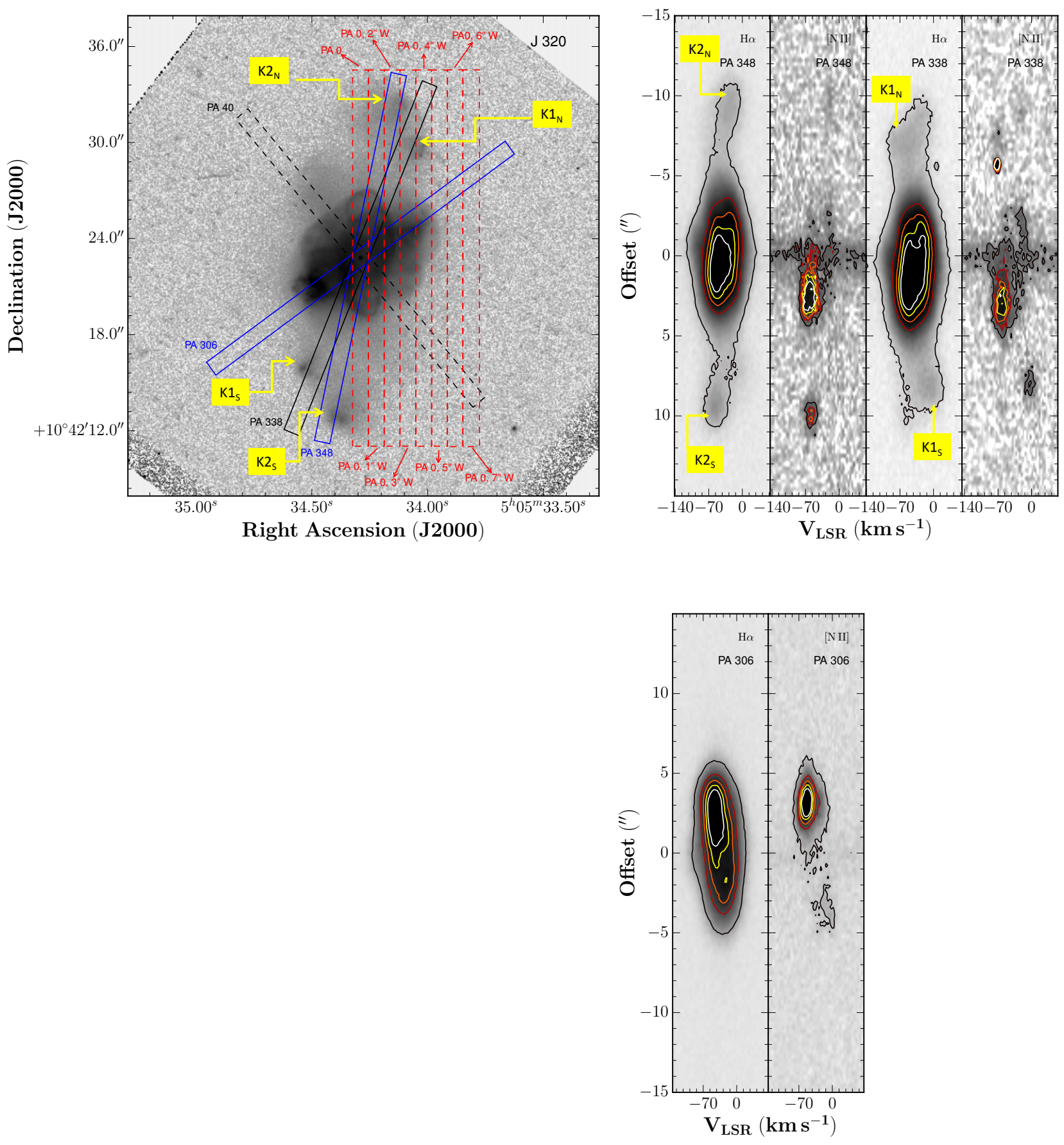

Figure 5. Same as Figure 1 for $\mathrm{J} 320$. In this case, the $H S T$ image was obtained using the F656N $\mathrm{H} \alpha$ filter.

of the slit at $\mathrm{PA}=82^{\circ}$ and $\mathrm{PA}=145^{\circ}$ are shown in Figure 6 The expansion velocity of the main nebular shell is not resolved. We estimate an upper limit of $7 \mathrm{~km} \mathrm{~s}^{-1}$ based on the line width.

The outflow-like features registered by our long-slit observations indeed reveal systemic velocities of $\pm 38.2 \mathrm{~km} \mathrm{~s}^{-1}$ at $\mathrm{PA}=$ $82^{\circ}$ and $\pm 47.7 \mathrm{~km} \mathrm{~s}^{-1}$ at $\mathrm{PA}=145^{\circ}$, which are faster than the expansion velocity of the nebular shell. The velocities here detected certainly imply that the arcs and loops seen in optical images have suffered a notable acceleration, most likely produced by the action of collimated outflows whose tips are not clearly identified in the PV maps. The outflows have projected distances from the CSPN of $1.18 \times 10^{17} \mathrm{~cm}(0.04 \mathrm{pc})$ and $1.07 \times 10^{17} \mathrm{~cm}(0.03 \mathrm{pc})$, respectively.

\subsection{1-37}

M 1-37 (PN G002.6-03.4) is the Starfish Twin of Hen 2-47 (Sahai] 2000). Its morphology, as shown by its $\mathrm{H} \alpha$ image (Figure 7), is 

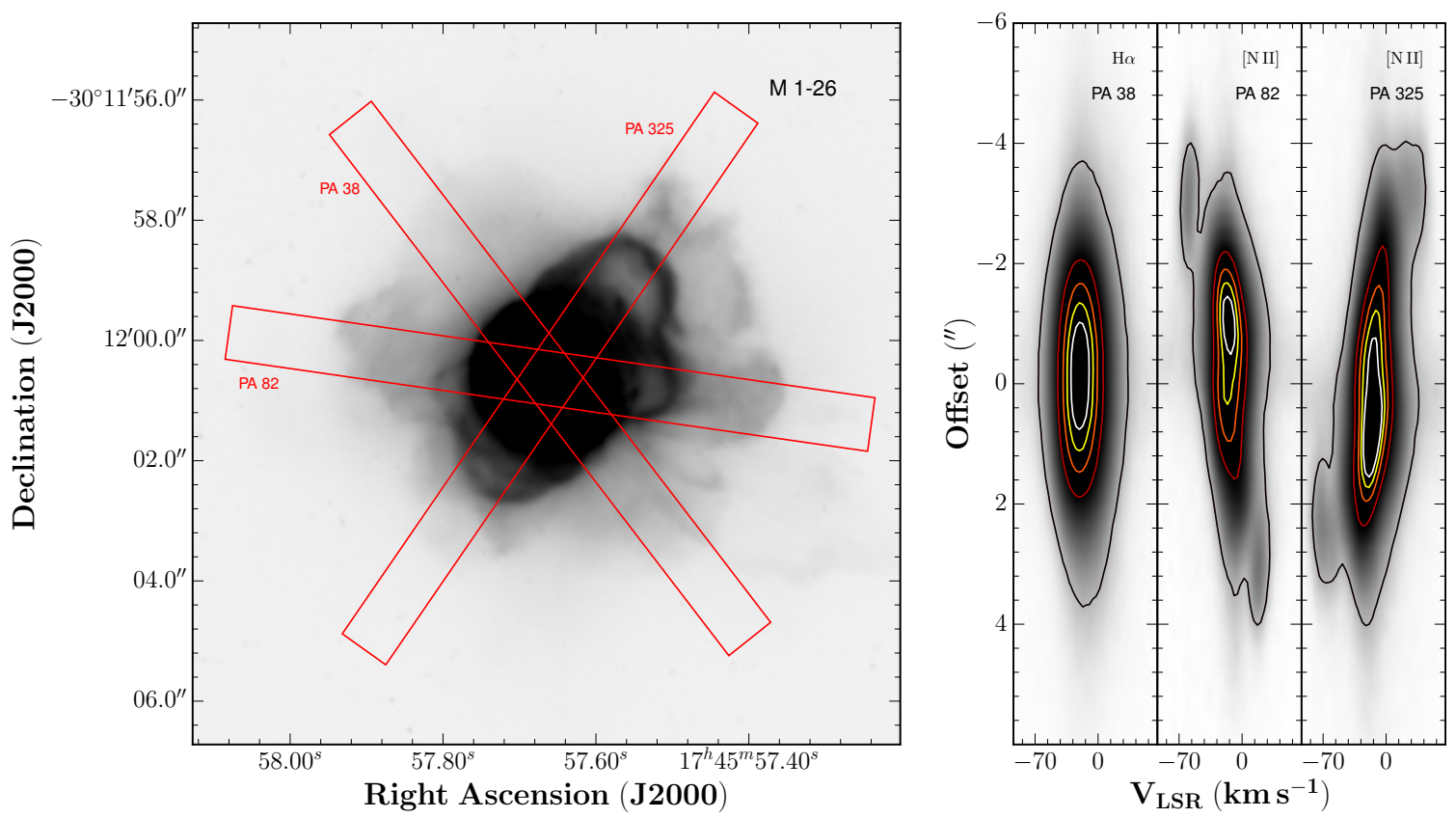

Figure 6. Same as Figure 1 for M 1-26. In this case, the HST image was obtained using the F656N H $\alpha$ filter.
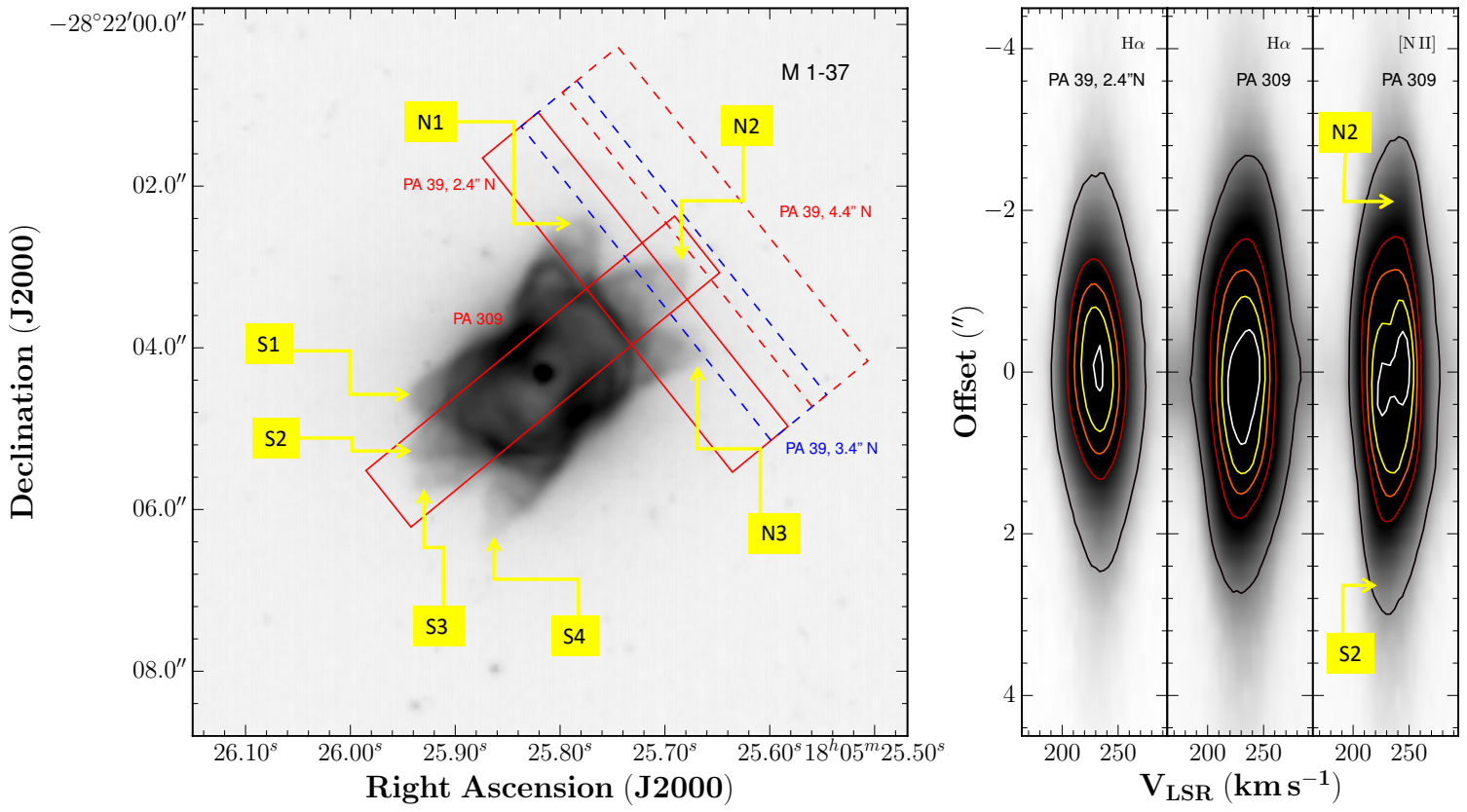

Figure 7. Same as Figure 1 for M 1-37. In this case, the HST image was obtained using the F656N H $\alpha$ filter.

multipolar, with seven $\mathrm{V}$-shaped lobes arranged along different directions from the highly distorted bipolar-shaped inner shell. Following Sahai (2000)'s naming convention for these lobes, we mark them from N1 to N3 at the North and S1 to S4 at the South in the $\mathrm{H} \alpha$ image in Figure 7 The main nebula is surrounded by a faint halo.

The kinematics of M 1-37 is investigated using a slit placed along its major axis $\left(\mathrm{PA}=129^{\circ}\right)$ and another one along $\mathrm{PA}=39^{\circ}$ offset from the central star to register the N1, N2, and N3 features (see the right panel of Fig. 77. A nebular expansion velocity of 11 $\mathrm{km} \mathrm{s}^{-1}$ is estimated from the PV maps of the slit at PA $=129^{\circ}$.

As for its twin, Hen 2-47, the V-shaped lobes do not show extremely narrow kinematical components, with a small systemic velocity for the pair N2-S2 of $v_{\mathrm{r}}^{\text {outflow }}= \pm 7.6 \mathrm{~km} \mathrm{~s}^{-1}$. These features have projected distances from the CSPN of $4.4 \times 10^{17} \mathrm{~cm}(0.14 \mathrm{pc})$. 


\subsection{1-66}

M 1-66 (PN G032.7-02.0) is a young PN whose rhomboidal inner shell is surrounded by an elliptical envelope. The first evidence of the presence of a bipolar outflow is found in the [N II] image presented by Manchado et al. (1996), later on described as a pair of collimated outflow oriented along the major nebular axis at $\mathrm{PA}=$ $131^{\circ}$ (Miranda, \& Guerrero 2006). These features are more clearly shown in the HST image presented in the left panel of Figure 8 (Sahai et al. 2011). A filament departs from the tip of the inner shell and extends up to two knots, with a morphology reminiscent of that described for the FLIERs of NGC 7009 (Balick et al. 1998). Soker (1997) proposed that the progenitor star of M 1-66 had interacted with a substellar companion.

We have placed a slit along the pair of collimated outflows $\left(\mathrm{PA}=131^{\circ}\right)$, as shown in the left panel of Figure 8 The $\mathrm{H} \alpha$ and $[\mathrm{N}$ II $] \lambda 6583 \mathrm{PV}$ maps are shown in the right panels of this figure. A lenticular featureless emission line is observed in the $\mathrm{H} \alpha \mathrm{PV}$ map, but the [N II] $\lambda 6583$ PV map reveals two knots in the equatorial regions of the nebula and two faint knots that protrude from the nebula. The expansion velocity of the nebular shell is $\simeq 20 \mathrm{~km} \mathrm{~s}^{-1}$.

The [N II] $\lambda 6583$ PV map confirm that M 1-66 indeed harbors a pair of collimated outflows, with very narrow kinematical components, although their semi-difference in radial velocity is small, $v_{\mathrm{r}}^{\text {outflow }}= \pm 7 \mathrm{~km} \mathrm{~s}^{-1}$. These features have projected distances from the CSPN of $3.3 \times 10^{17} \mathrm{~cm}(0.106 \mathrm{pc})$.

\subsection{2-40}

M 2-40 (PN G24.1+03.8) is a multiple-shell PN with a detachedhalo interacting with the interstellar medium (Guerrero et al.|1998). The nebula was classified as elliptical using ground-based images and the same high-dispersion long-slit data used here (Stanghellini et al. 2002), but an HST H $\alpha$ image suggests it is bipolar with closed lobes and major axis along $\mathrm{PA}=80^{\circ}$ (Sahai et al. 2011). Two knots protrude from the main nebular shell along the major axis and produce bow-shock-like features.

The kinematics of M 2-40 has been investigated using a slit along the major axis at $\mathrm{PA}=80^{\circ}$ (Figure 9). The $\mathrm{H} \alpha$ and [N II] $\lambda 6583$ PV maps reveal a knotty inner shell with an ellipsoidal rather than bipolar structure. Indeed, Guerrero et al. (1998) interpreted this same PV map as evidence for an ellipsoidal shell. There is additional faint emission detected at a radial distance of $\simeq 10^{\prime \prime}$ from the CSPN (not shown in the PV maps) that corresponds to an arc of enhanced emission resulting from the interaction of the halo with the interstellar medium (Guerrero et al. 1998). The nebula has an expansion velocity $\simeq 17.6 \mathrm{~km} \mathrm{~s}^{-1}$, as derived from the split of the $[\mathrm{N}$ II] emission line at the location of the CSPN.

The pair of knots outside the main nebular shell are detected in these PV maps and confirmed to be collimated outflows, although their systemic velocity is not particularly high, $v_{\mathrm{r}}^{\text {outflow }}= \pm 16.0$ $\mathrm{km} \mathrm{s}^{-1}$. This pair of outflows have projected distances from the $\mathrm{CSPN} \sim 3.8 \times 10^{17} \mathrm{~cm}(0.12 \mathrm{pc})$.

\subsection{3-1}

M3-1 (PN G242.6-11.6) is a PN whose morphology has been classified as point-symmetric (Gonçalves et al. 2001) based on the $\mathrm{H} \alpha+[\mathrm{N} \mathrm{II}]$ image and $\mathrm{H} \alpha+[\mathrm{N}$ II $] /[\mathrm{O}$ III $]$ ratio map presented by Corradi et al. (1996). The HST [N II] image (Figure 10-left) reveals an intricate morphology, with an S-shaped point-symmetric low-ionization structure suggestive of a precessing collimated outflow and a filamentary disk-like structure at the central nebular regions. Bear, \& Soker (2017) suggested that M3-1 maybe shaped by a triple stellar progenitor. It definitely harbors at least a closebinary system with a very short period (Jones et al. 2019).

The kinematics of M 3-1 has been investigated using long-slit spectra obtained at three slit positions with $\mathrm{PA}=72^{\circ}$ (the disk-like structure) and $318^{\circ}$ and $358^{\circ}$ (the S-shaped structure). The three [N II] $\lambda 6583$ PV maps reveal a knotty main body (indicated by the yellow contours in the right panels of Fig. 10, embedded within a shell of diffuse emission. This shell of diffuse emission dominates the $\mathrm{H} \alpha$ echellograms, as illustrated in the $\mathrm{H} \alpha \mathrm{PV}$ map along $\mathrm{PA}=$ $318^{\circ}$ displayed in the right panel of Figure 10 . The nebula has an expansion velocity $\simeq 24.5 \mathrm{~km} \mathrm{~s}^{-1}$.

The S-shaped structure is clearly detected in the PV maps along $\mathrm{PA}=318^{\circ}$ and $\mathrm{PA}=358^{\circ}$ as pairs of resolved knots located outside the main body, i.e., they are collimated outflows. In addition, there is one small knot at $\mathrm{PA}=318^{\circ}$ between the main nebular shell and the top knot which correspond to a filament in M 3-1. The systemic velocity of the collimated outflows along PA $=358^{\circ}$ is $\pm 14.9 \mathrm{~km} \mathrm{~s}^{-1}$ and for those along $\mathrm{PA}=318^{\circ}$ is $\pm 13.2 \mathrm{~km} \mathrm{~s}^{-1}$. The tip of this precessing jet-like structure has projected distances from the CSPN of $\sim 8.3 \times 10^{17} \mathrm{~cm}(0.27 \mathrm{pc})$.

\subsection{NGC 6210}

NGC 6210 (PN G043.1+37.7) has a complex shape, described to be amorphous or irregular (Aller et al. 1970, Balick 1987, Icke et al. 1989) with a general elliptical structure (Stanghellini et al. 2006, Soker 2016). This nebula presents a very bright central region with filaments and knots (Balick et al. 1987), surrounded by a pair of collimated outflows with a point-symmetric morphology (Phillips, \& Cuesta 1996, Guerrero et al. 2013). These jets are younger that the main shells (Gonçalves et al. 2001). The nebula is surrounded by a faint halo-like structure (Pottasch et al.2009).

The kinematics of the complex nebula NGC 6210 was investigated using two slits. The first one $\left(\mathrm{PA}=156^{\circ}\right)$ along a knot located far from the CSPN labeled as $\mathrm{C}$ and also along an extensive outflow with a S-shape fainter than $\mathrm{C}$. The second one $\left(\mathrm{PA}=132^{\circ}\right)$ oriented along two knot-like features labeled as A and B in Figure 11 The $\mathrm{H} \alpha \mathrm{PV}$ maps for both slits show a lenticular shape, while the [N II] $\lambda 6583$ PV maps show an irregular shape with knots surrounded by a faint shell. The expansion velocity of NGC 6210 is found to be $\simeq 34.2 \mathrm{~km} \mathrm{~s}^{-1}$.

The systemic velocity of the collimated outflows along PA = $132^{\circ}$ is $\pm 19.5 \mathrm{~km} \mathrm{~s}^{-1}$ for outflow A, $\pm 30.6 \mathrm{~km} \mathrm{~s}^{-1}$ for outflow B and, for the slit along $\mathrm{PA}=156^{\circ}, \pm 29.4 \mathrm{~km} \mathrm{~s}^{-1}$ for outflow $\mathrm{C}$. The three outflows labeled as $\mathrm{A}, \mathrm{B}$ and $\mathrm{C}$ have projected distances from the CSPN of $\sim 2.6 \times 10^{17} \mathrm{~cm}(0.08 \mathrm{pc}), \sim 1.4 \times 10^{17} \mathrm{~cm}(0.04 \mathrm{pc})$, and $\sim 5.4 \times 10^{17} \mathrm{~cm}(0.17 \mathrm{pc})$, respectively.

\subsection{NGC 6741}

NGC 6741 (PN G033.8-02.6) has been described as a bright ellipsoidal nebulae with faint inner structures and filaments based on ground-based images (Curtis et al. 1918, Zuckerman, \& Aller 1986, Stanghellini et al. 1993, Sabbadin et al. 2005). The HST [N II] image of this PN reveals additional faint emission outside the main nebular shell and outflow-like features along its major axis (Figure 12.

To investigate the kinematics of NGC 6741, we have placed 

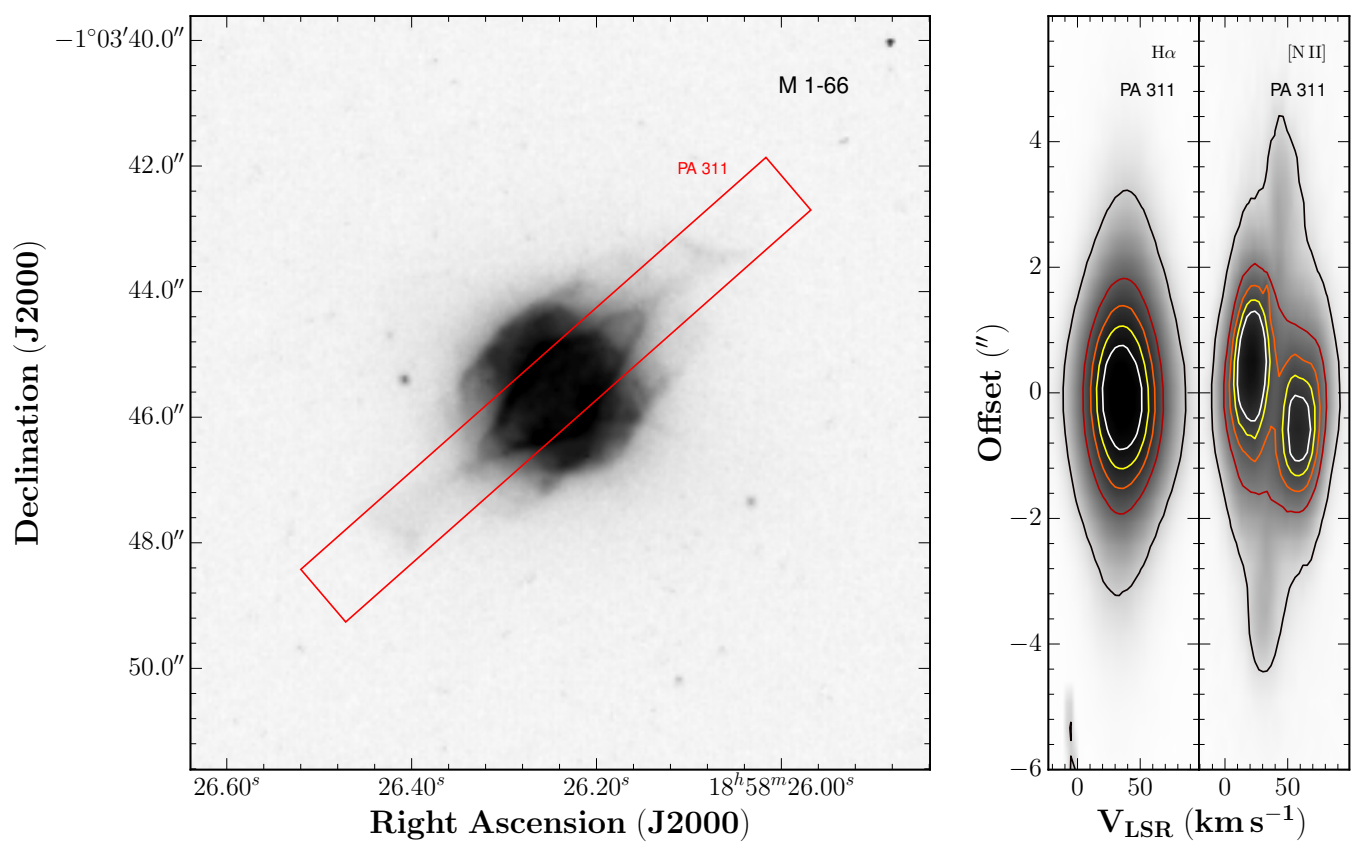

Figure 8. HST WFPC2 F656N H $\alpha$ image (left) and $\mathrm{H} \alpha$ and [N II] $\lambda 6583 \mathrm{PV}$ maps derived from WHT UES echelle spectra (right) of M 1-66.
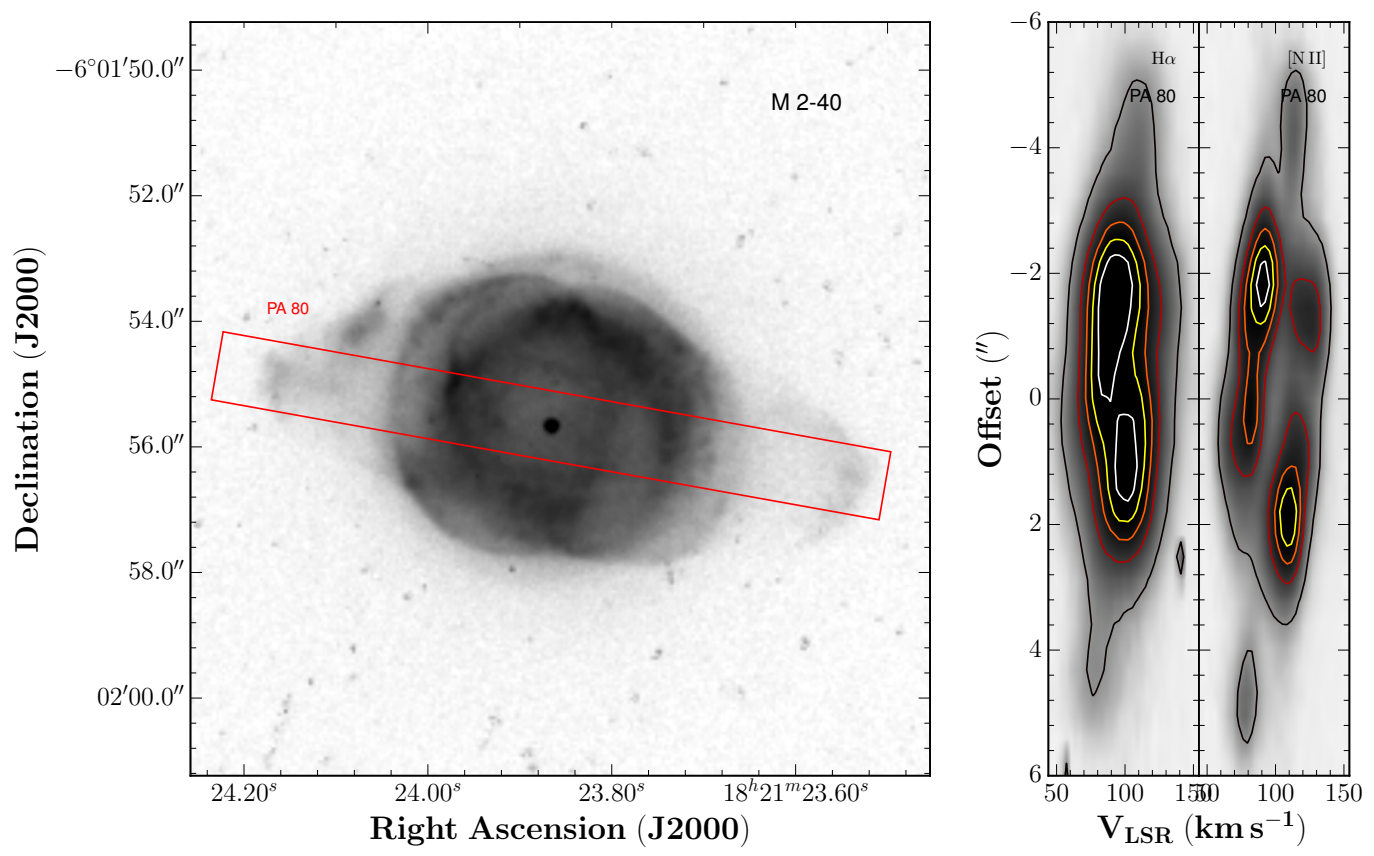

Figure 9. Same as Figure 8 for M 2-40.

a slit along the outflow-like feature $\left(\mathrm{PA}=77^{\circ}\right)$ and two along the main nebular shell at $\mathrm{PA}=18^{\circ}$ and $122^{\circ}$. All $[\mathrm{N} \mathrm{II}] \lambda 6583 \mathrm{PV}$ maps are indicative of a shell-like structure (Fig.12,right), which is otherwise not well resolved in the $\mathrm{H} \alpha \mathrm{PV}$ maps, with an S-shaped morphology along $\mathrm{PA}=77^{\circ}$. In particular, the slit at $\mathrm{PA}=18^{\circ}$ along the minor axis reveals a toroidal shape, which in conjunction with the PV maps at $\mathrm{PA}=18^{\circ}$ and $122^{\circ}$ imply that the main nebular shell is tilted along the latter direction. Its nebular expansion velocity is $\simeq 23.4 \mathrm{~km} \mathrm{~s}^{-1}$.

The PV maps along the major axis reveal the presence of a collimated outflow mostly in the [N II] $\lambda 6583$ emission line. These are labeled A, B, and C in Figure 12 The systemic velocity of features $\mathrm{A}$ and $\mathrm{B}$ is $\pm 22.7 \mathrm{~km} \mathrm{~s}^{-1}$, but only $+7.3 \mathrm{~km} \mathrm{~s}^{-1}$ for feature $\mathrm{C}$, suggesting a steep velocity gradient. The pair of outflows A-B have projected distances from the CSPN of $\sim 3.9 \times 10^{17} \mathrm{~cm}(0.13$ $\mathrm{pc})$, whereas outflow $\mathrm{C}$ is found at a projected radial distance of $\sim 3.1 \times 10^{17} \mathrm{~cm}(0.10 \mathrm{pc})$. 

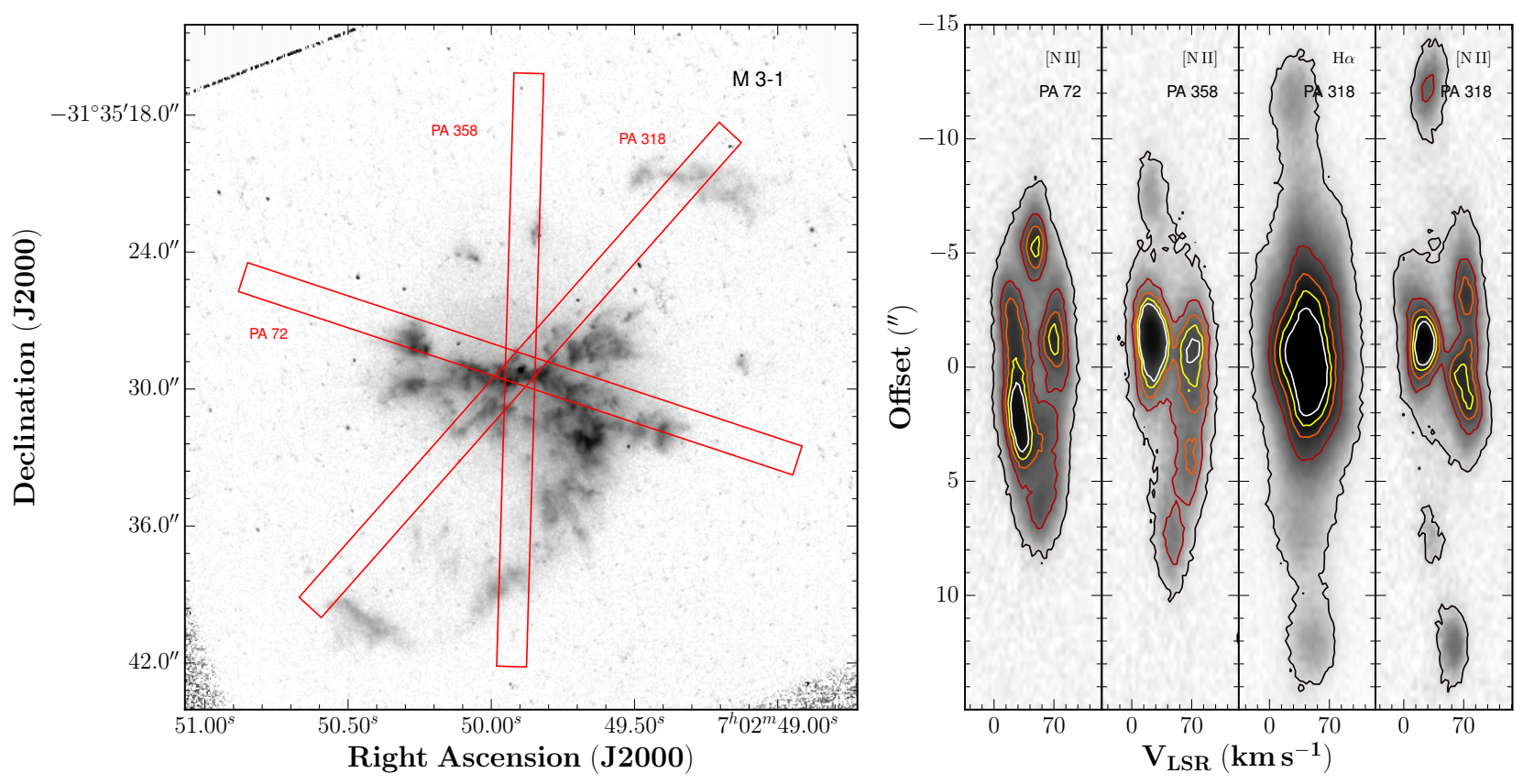

Figure 10. Same as Figure 1 for M3-1.
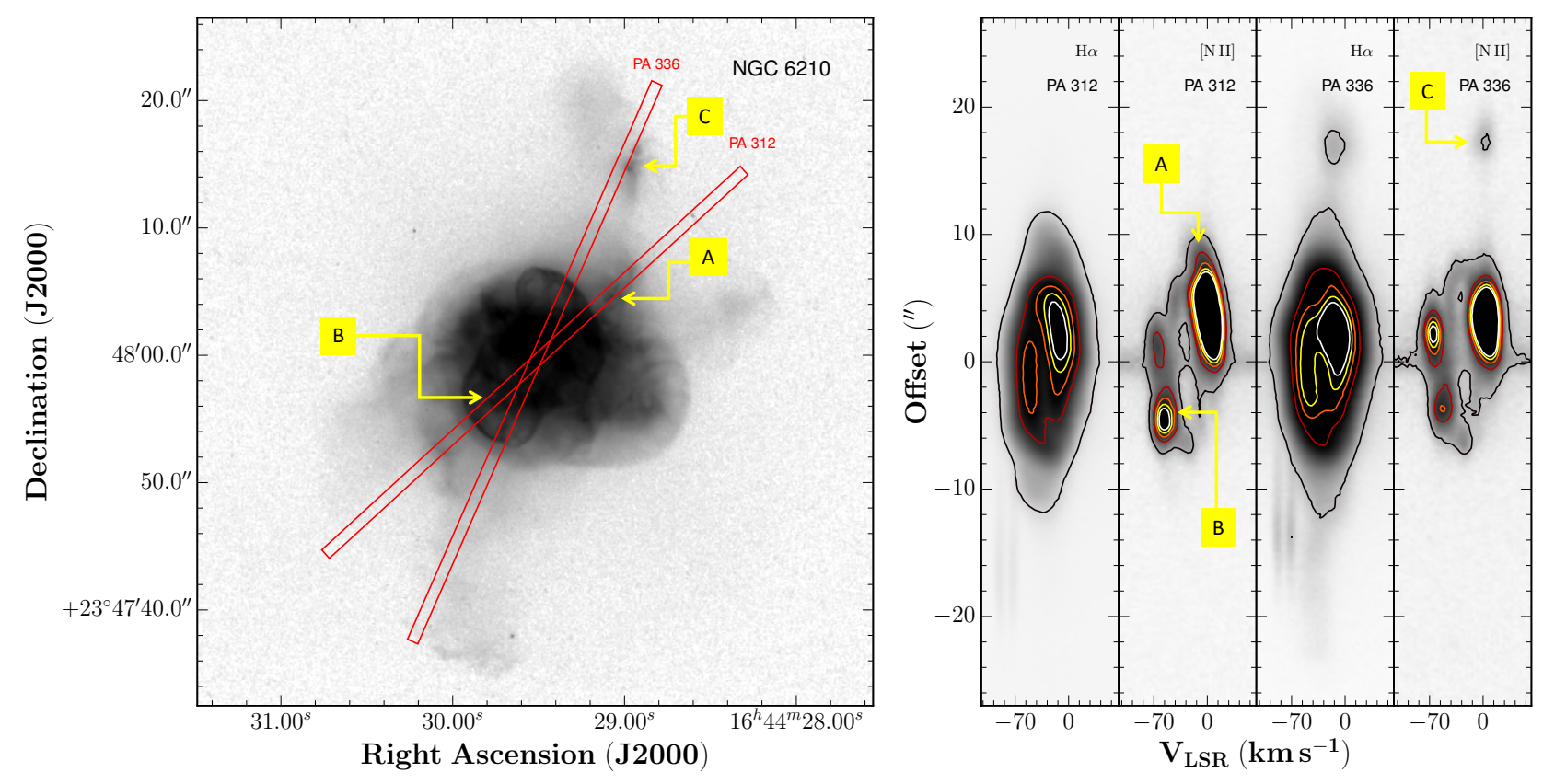

Figure 11. Same as Figure 1 for NGC 6210.

\section{DISCUSSION}

Narrow-band images of a significant fraction of PNe show lowionization morphological features that are suggestive of the presence or action of fast collimated outflows. Here we have selected a sample of 12 of those PNe and obtained kinematical information by means of long-slit high-dispersion echelle spectra across morphological features suspected to be associated with fast collimated outflows. The nature of these features, summarized in the seventh column of Table 2, can be divided into two main groups.

In the first group we include those PNe where indeed the presence of collimated outflows is confirmed, namely Hen 2-429, the outermost C-D features of IC 4776, J 320, M 1-66, M 2-40, M3-1, NGC 6210, and NGC 6741. In all those cases, the low-ionization morphological features can be associated with narrow kinematical components well resolved spatial and kinematically from the 

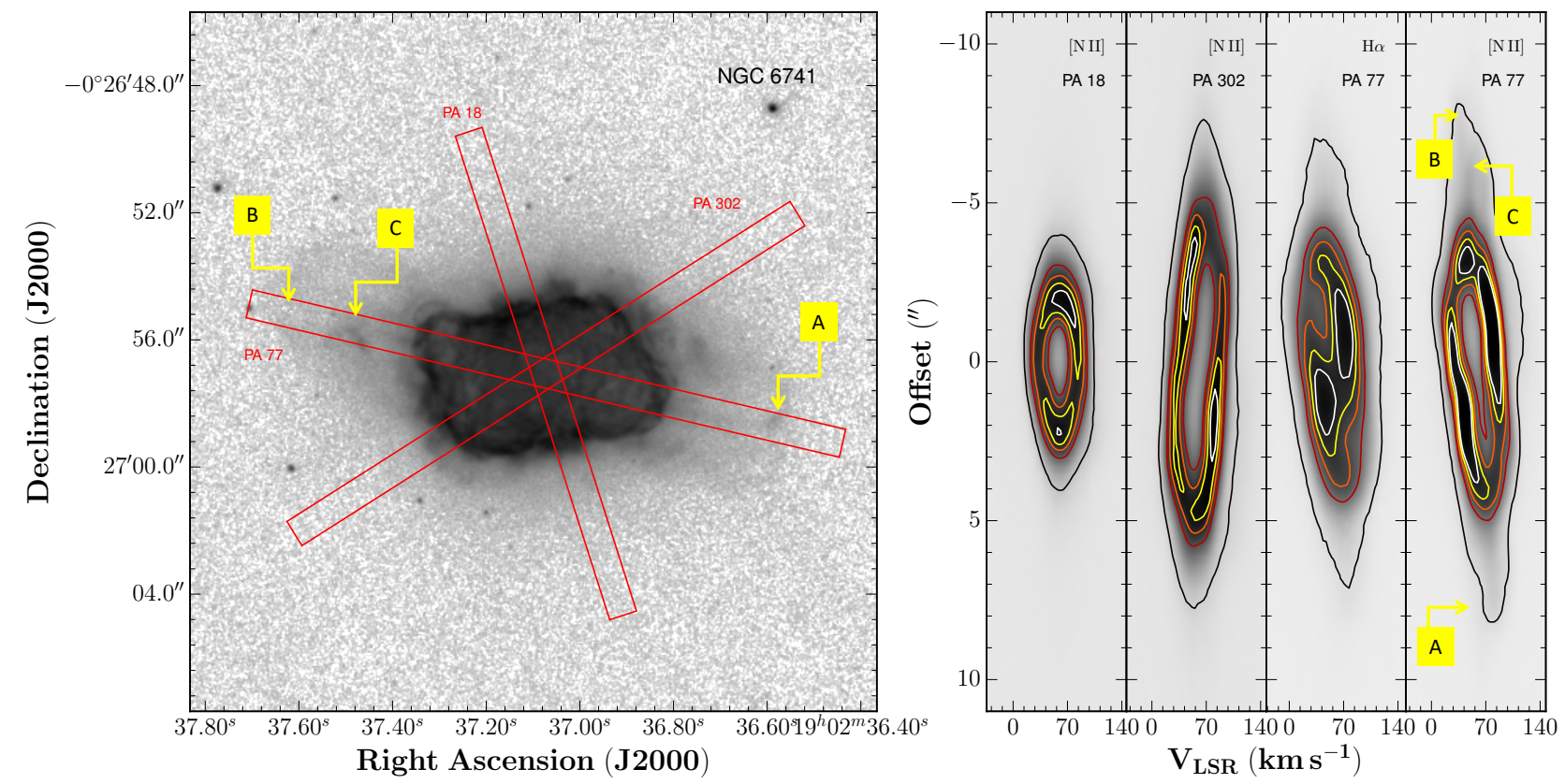

Figure 12. Same as Figure 1 for NGC 6741.

main nebular shells. We note that, for the outermost C-D features of IC 4776, deeper narrow-band images and high-dispersion spectra may help eludicate whether these are real collimated outflows or a second pair of bipolar lobes. Cases of particular interest are those of M 1-66, M 2-40, and NGC 6741, whose collimated outflows can be described as jet-like features in the PV maps. Similarly, the collimated ouflows of Hen 2-429, M 3-1, and NGC 6210 are suggestive of precessing jets, whereas those of J 320 can be described as multiple bipolar ejections (e.g., Bipolar Rotating Episodic Ejections, BRETs, López et al. 1995).

In the second group we include those PNe where the presence of fast collimated outflows cannot be confirmed kinematically, namely Hen 2-47, Hen 2-115, the innermost A-B features of IC 4776, M 1-26 and M 1-37. The innermost A-B features of IC 4776 seem to be located at the tip of bipolar lobes, closing a tilted hourglass structure in the PV map. The other four PNe share interesting morphological properties, with V-shaped lobes in the Starfish Twins Hen 2-47 and M 1-37, and in Hen 2-115 and M 126. The PV maps along these features imply kinematics differing from those of their main nebular shells, particularly for Hen 2-47 and M 1-26, with notably higher expansion velocities. These perturbations in the kinematics, together with the peculiar morphology of these features, may result from the action of stellar outflows arising at the end of the AGB phase (Sahai, \& Trauger 1998) that we cannot detect either because they are too tenuous or because they are not ionized, as the molecular outflows reported in NGC 7027 (Cox et al. 2002; Huang et al. 2010). Apparently, these outflows have not been able to pierce through the nebular envelope, which has retained the linear momentum from the outflow and therefore experienced a notable acceleration. This points out to "light" outflows, defined to have densities much lower than that of the slow AGB wind (Akashi, \& Soker 2008).

The velocity signature of these $\mathrm{V}$-shaped morphological features can be investigated using their apparent opening angle to esti- mate the Mach number of the ejections producing these lobes. This is referred as the Mach angle $\alpha$, and can be related to the Mach number $M$ as

$\sin \alpha=\frac{1}{M}$

where $M$ is defined as the ratio $v / c_{\mathrm{s}}$ between the velocity of the Mach front $v$ and the isothermal sound speed of ionized gas $c_{\mathrm{s}} \approx 10 \mathrm{~km} \mathrm{~s}^{-1}$. The Mach angles derived for Hen 2-47, Hen 2115 and $\mathrm{M} 1-37$ are $27^{\circ}, 23.5^{\circ}$, and $25^{\circ}$, which correspond to very similar Mach numbers of 2.20, 2.50, and 2.36, respectively. As for M 1-26, no Mach number has been derived because its lobes do not have well defined V-shape. For inclination angles with the plane of the sky smaller than $45^{\circ}$, these expansion velocities can be added quadratically with the radial velocities listed in Table 2 to derive the space velocity of the ejections that produced these V-shaped lobes with an uncertainty caused by the unknown inclination angle smaller than $10 \%$. These are $35 \mathrm{~km} \mathrm{~s}^{-1}, 25 \mathrm{~km} \mathrm{~s}^{-1}$, and $24 \mathrm{~km} \mathrm{~s}^{-1}$ for Hen 2-47, Hen 2-115 and M 1-37, respectively. Therefore, the V-shaped lobes of these sources expand at velocities $\sim 2-3$ times that of their (slow) main nebular shells (see Table 2).

It is interesting to note the similar morphology of the V-shaped structure along the major axis and direction of the collimated outflow of Hen 2-429 and particularly M 1-66. This may indicate that the collimated outflows in these two PNe had sufficient momentum to break through the nebular envelope from the AGB. In this sense, the collimated outflows in NGC 6543 and NGC 7009, arising from V-shaped features at the tip of the major axis of their inner shells, can be interpreted as much more evolved versions of these interactions. It can thus be envisaged an evolutionary sequence where collimated outflows start piercing through the nebular envelope like in Hen 2-47, Hen 2-115, M 1-26, and M 1-37, then break outside this envelope like in Hen 2-429 and M 1-66, and finally emerge like in NGC 6543 and NGC 7009. The kinematical ages of PNe with and 
without collimated outflows have similar ranges, and thus they do not support this interpretation, although uncertainties in their distances and assessment of their true ages through the kinematic age may hinder such trend (Schönberner et al. 2005). There is additional evidence that the four PNe without clear collimated outflows belong to a different group from the other PNe in our sample. All of them can be classified as very low excitation $\mathrm{PNe}([\mathrm{O} \mathrm{III}] / \mathrm{H} \alpha \lesssim 1)$ with low effective temperature CSPNe (Sahai, \& Trauger 1998, Sahai et al. 2011), which can be interpreted as a sign of either an early evolutionary phase or slow post-AGB evolution as expected for smaller mass progenitors (Miller Bertolami 2016). It is interesting to note that these sources also exhibit the smallest nebular expansion velocities among our sample of PNe (Tab.2, which would also place them at an early evolutionary phase (Richer et al. 2008 2010) according to theoretical models of nebular evolution (Jacob et al. 2013), but that can also be explained if their progenitors had low masses (Pereyra et al. 2016).

\section{SUMMARY}

We present a spatio-kinematical investigation of a sample of twelve PNe suspected to have collimated outflows using archival narrowband images and high-dispersion long-slit echelle spectra. Collimated outflows, which can be described as distinct narrow kinematical components with notable velocity variations with respect to the main nebular shells associated with compact knots and linear or precessing jet-like features, are confirmed in seven sources, with one additional possible detection in IC 4776. In all other cases, the nature of the possible collimated outflows is not confirmed or even rejected, as the case of the innermost A-B components of IC 4776, which can be associated with a pair of bipolar lobes. The morphology and kinematics of the four sources where collimated outflows are not confirmed, namely the Starfish Twins Hen 2-47 and M 137, and Hen 2-115 and M 1-26, are indicative of the action of collimated outflows which are not detected in optical images and spectra. These PNe appear to be at an early evolutionary phase or descend from lower mass progenitors than the $\mathrm{PNe}$ in our sample with unambiguous collimated outflows.

\section{ACKNOWLEDGMENTS}

JSR-G and MAG acknowledge support of the grants AYA 201457280-P and PGC2018-102184-B-I00, co-funded with FEDER funds. SDP acknowledges financial support from the Spanish Ministerio de Economía y Competitividad under grants AYA2013 47742-C4-1-P and AYA2016 79724-C4-4-P, from Junta de Andalucía Excellence Project PEX2011 FQM-7058. JAT and MAG are also funded by UNAM DGAPA PAPIIT project IA100318. LFM acknowledges partial support by grant AYA201784390-C2-R, co-funded with FEDER funds. JSR-G, MAG, LFM, and SDP acknowledge financial support from the State Agency for Research of the Spanish MCIU through the "Center of Excellence Severo Ochoa" award for the Instituto de Astrofísica de Andalucía (SEV-2017-0709). We thank Dr. Laurence Sabin for a thorough reading of the manuscript and her valuable comments that helped us to improve it.

Based on observations made with the NASA/ESA Hubble Space Telescope, and obtained from the Hubble Legacy Archive, which is a collaboration between the Space Telescope Science Institute (STScI/NASA), the Space Telescope European Coordinat- ing Facility (STECF/ ESA) and the Canadian Astronomy Data Centre (CADC/NRC/CSA). This research has made also use of the NASA's Astrophysics Data System.

This research made use of Python (http://www.python.org) and IPython (Pérez, \& Granger 2007), APLpy (Robitaille, \& Bressert 2012), Numpy (Van Der Walt et al. 2011), Pandas (McKinney 2010), and Matplotlib (Hunter 2007), a suite of open-source Python modules that provides a framework for creating scientific plots. This research made use of Astropy, a community-developed core Python package for Astronomy (Astropy Collaboration et al. 2013). The Astropy web site is http://www . astropy.org

\section{REFERENCES}

Akashi, M., \& Soker, N. 2008, MNRAS, 391, 1063

Aller L.H., Czyzak S.J., \& Buerger E.G. 1970, ApJ, 162, 783

Astropy Collaboration, Robitaille, T. P, Tollerud, E. J. et al. 2013, A\&A, 558, A33

Balick, B. 1987, ApJ, 94, 671

Balick, B., Alexander, J., Hajian, A.R., Terzian, Y., Perinotto, M., \& Patriarchi, P. 1998, ApJ, 116, 360

Balick, B., Gonzalez, G., Frank, A., \& Jacoby G. 1992, ApJ, 392, 582

Balick, B., Huarte-Espinosa, M., Frank, A., Gomez, T., Alcolea, J., Corradi, R.L.M., \& Vinković D. 2013, ApJ, 772, 20

Balick, B., Riera, A., Raga, A., Kwitter, K. B., \& Velázquez, P.F. 2014, ApJ, 795, 83

Beaulieu, S.F., Dopita, M.A., \& Freeman, K.C. 1999, ApJ, 515, 610

Bear E., \& Soker N. 2017, ApJ, 837, L10

Bianchi, L. 1992, A\&A, 260, 314

Campbell, W.W., \& Moore, J.H. 1917, PASP, 29, 56

Cliffe, J.A., Frank, A., Livio, M., \& Jones, T.W. 1995, ApJ, 447, L49

Clyne, N., Akras, S., Steffen, W., Redman, M.P., Gonçalves, D.R., \& Harvey E. 2015, A\&A, 582, A60

Contreras, M.E., Vázquez, R., Miranda, L.F., Olguín, L., Zavala, S., \& Ayala, S. 2010, ApJ, 139, 1426

Corradi, R.L.M., Gonçalves, D.R., Villaver, E., Mampaso, A., Perinotto, M., Schwarz, H.E., \& Zanin, C. 2000, ApJ, 535, 823

Corradi, R.L.M., Manso, R., Mampaso, A., \& Schwarz, H.E. 1996, A\&A, 313, 913

Cox, P., Huggins, P.J., Maillard, J.-P., Habart, E., Morisset, C., Bachiller, R., \& Forveille T. 2002, A\&A, 384, 603

Curtis, H.D. 1918, Publications of Lick Observatory, 13, 55

Durand, S., Acker, A., \& Zijlstra A. 1998, A\&AS, 132, 13

Frew, D.J., Parker, Q.A., \& Bojičić, I.S. 2016, MNRAS, 455, 1459

Gonçalves, D.R., Corradi, R.L.M., \& Mampaso, A. 2001, ApJ, 547,302

Górny, S.K., Stasińska, G., \& Tylenda, R. 1997, A\&A, 318, 256

Guerrero, M.A., Rechy-García, \& Ortiz, R. 2019, ApJ, submitted Guerrero, M.A., Villaver, E., \& Manchado A. 1998, ApJ, 507, 889 Guerrero, M.A., Vázquez, R., \& López, J.A. 1999, ApJ, 117, 967

Guerrero, M.A., Miranda, L.F., Riera, A., et al. 2008, ApJ, 683, 272

Guerrero, M.A., Toalá, J.A., Medina, J.J., et al. 2013, A\&A, 557, A121

Harman, D.J., Bryce, M., López, J.A., Meaburn, J., \& Holloway, A.J. 2004, MNRAS, 348, 1047 


\section{4}

Rechy-García et al.

Haro-Corzo, S.A.R., Velázquez, P.F., Raga, A.C., Riera, A., \& Kajdic P. 2009, ApJ, 703, L18

Huang, Z.-Y., Hasegawa, T. I., Dinh-V-Trung, et al. 2010, ApJ, 722,273

Hunter, J.D. 2007, Computing In Science \& Engineering, 9, 90

Icke, V., Preston, H.L., \& Balick, B. 1989, ApJ, 97, 462

Jacob R., Schönberner D., Steffen M., 2013, A\&A, 558, A78

Jonckheere, R. 1916, The Observatory, 39, 134

Jones, D., Boffin, H.M.J., Sowicka, P. et al. 2019, MNRAS, 482, L75

López, J.A. 1997, in IAU Symp 180, Planetary Nebulae, ed. H.J. Habing \& H.J.G.L.M. Lamers (Dordrecht: Kluwer), 197

López, J.A., Vázquez, R., \& Rodríguez, L.F. 1995, ApJ, 455, L63 Maciel, W.J., \& Dutra, C.M. 1992, A\&A, 262, 271

Manchado, A., Guerrero, M.A., Stanghellini, L., \& Serra-Ricart, M. 1996, The IAC Morphological Catalog of Northern Galactic Planetary Nebulae, IAC Publications

Mellema, G., Eulderink, F., \& Icke, V. 1991, A\&A, 252, 718

McKinney, W. 2010, Proceedings of the 9th Python in Science Conference, ed. S. van der Walt \& J. Millman, 51

Miller Bertolami, M.M. 2016, A\&A, 588, A25

Miranda, L.F. 1995, A\&A, 304, 531

Miranda, L.F., \& Guerrero, M.A. 2006, Planetary Nebulae in our Galaxy and Beyond, 234, 461

Ortolani, S., \& Sabbadin, F. 1985, A\&AS, 62, 17

Pereyra, M., López, J.A., \& Richer, M.G. 2016, ApJ, 151, 53

Pérez, F., \& Granger, B.E. 2007, Computing In Science \& Engineering, 9, 21

Phillips, J.P., \& Cuesta, L. 1996, ApJ, 111, 1227

Pottasch, S.R., Bernard-Salas, J., \& Roellig T.L. 2009, A\&A, 499, 249

Rechy-García, J.S., Velázquez, P.F., Pena, M., \& Raga, A.C. 2017, MNRAS, 464, 2318

Richer, M.G., López, J.A., García-Díaz, M.T., Clark, D.M., Pereyra, M., \& Díaz-Méndez, E. 2010, ApJ, 716, 857

Richer, M.G., López, J.A., Pereyra, M., Riesgo, H., García-Díaz, M.T., \& Báez, S.-H. 2008, ApJ, 689, 203

Richer, M.G., Suárez, G., López, J.A., \& García Díaz, M.T. 2017, ApJ, 153, 140

Robitaille, T., \& Bressert, E. 2012, APLpy: Astronomical Plotting Library in Python, Astrophysics Source Code Library

Sabbadin, F., Benetti, S., Cappellaro, E., Ragazzoni, R., \& Turatto, M., 2005, A\&A, 436, 549

Sahai, R., \& Trauger, J.T. 1998, ApJ, 116, 1357

Sahai, R. 2000, ApJ, 537, L43

Sahai, R., Morris, M.R., \& Villar G.G. 2011, ApJ, 141, 134

Schneider, S.E., Terzian, Y., Purgathofer, A., \& Perinotto, M. 1983, ApJs, 52, 399

Schwarz, H.E., 1992, A\&A, 264, L1

Schwarz, H.E., Aspin, C., Corradi, R.L.M., \& Reipurth B. 1997, aap, 319, 267

Schönberner, D., Jacob, R., \& Steffen, M. 2005, A\&A, 441, 573

Soker, N. 1992, ApJ, 389, 628

Soker, N. 1997, ApJs, 112, 487

Soker, N. 2016, MNRAS, 455, 1584

Sowicka, P., Jones, D., Corradi, R.L.M., Wesson, R., GarcíaRojas, J., Santander-García, M., Boffin, H.M.J., \& RodríguezGil, P. 2017, MNRAS, 471, 3529

Stanghellini, L., Corradi, R.L.M., \& Schwarz, H.E. 1993, A\&A, 276, 463

Stanghellini, L., Villaver, E., Manchado, A., \& Guerrero, M.A. 2002, ApJ, 576, 285
Stanghellini, L., Guerrero, M.A., Cunha, K., Manchado, A., \& Villaver, E. 2006, ApJ, 651, 898

Uscanga, L., Velázquez, P.F., Esquivel, A., Raga, A.C., Boumis, P., \& Cantó, J. 2014, MNRAS, 442, 3162

Van Der Walt, S., Colbert, S.C., \& Varoquaux, G. 2011, ArXiv e-prints

Wright, W.H. 1918, Publ. Lick Obs., 13, Part VI

Zuckerman, B., \& Aller, L.H. 1986, ApJ, 301, 772

This paper has been typeset from a $\mathrm{T}_{\mathrm{E}} \mathrm{X} / \mathrm{LTT}_{\mathrm{E}} \mathrm{X}$ file prepared by the author. 